\title{
Assessing the Pharmacological and Therapeutic Efficacy of Traditional Chinese Medicine Liangxue Tongyu Prescription for Intracerebral Hemorrhagic Stroke in Neurological Disease Models
}

\section{OPEN ACCESS}

Edited by:

Aiping Lu,

Hong Kong Baptist University,

Hong Kong

Reviewed by:

Adam Matkowski.

Wroclaw Medical University, Poland

Joe Tauskela,

National Research Council Canada

(NRC-CNRC), Canada

${ }^{*}$ Correspondence:

Lian Yin

njxlyl2002@163.com

Guochun Li

liguochuncn@126.com

${ }^{\dagger}$ These authors share first authorship

Specialty section: This article was submitted to

Ethnopharmacology,

a section of the journal

Frontiers in Pharmacology

Received: 14 December 2017

Accepted: 26 September 2018

Published: 06 November 2018

Citation:

Li X, Huang $X$, Tang $Y$, Zhao F,

Cao Y, Yin L and Li G (2018)

Assessing the Pharmacological and

Therapeutic Efficacy of Traditional

Chinese Medicine Liangxue Tongyu

Prescription for Intracerebral

Hemorrhagic Stroke in Neurological

Disease Models.

Front. Pharmacol. 9:1169.

doi: 10.3389/fphar.2018.01169

\begin{abstract}
Xun $\mathrm{Li}^{1+}$, Xi Huang ${ }^{2 \dagger}$, Yuanlin Tang ${ }^{1}$, Fangli Zhao ${ }^{1}$, Yanmei Cao ${ }^{1}$, Lian Yin ${ }^{1 *}$ and Guochun Li ${ }^{3 *}$

${ }^{1}$ College of Pharmacy, Nanjing University of Chinese Medicine, Nanjing, China, ${ }^{2}$ Jiangsu Wujin Vocational School, Changzhou, China, ${ }^{3}$ College of Preclinical Medical, Nanjing University of Chinese Medicine, Nanjing, China
\end{abstract}

Intracerebral hemorrhage is a fatal subtype of stroke, with crucial impact on public health. Surgical removal of the hematoma as an early-stage treatment for $\mathrm{ICH}$ can't improve long-term prognosis remarkably. Liangxue tongyu prescription (LP), a Traditional Chinese Medicine (TCM) formula, includes eight ingredients and has been used to treat $\mathrm{ICH}$ in the clinical. In the study, we elucidated the pharmacological efficacy and therapeutic efficacy of LP to dissect the mechanism of LP against ICH via network analysis and experimental validation. First, we discovered 34 potential compounds and 146 corresponding targets in LP based on network prediction. 24 signal pathway were obtained by the Clue Go assay based on potential compounds in LP against $\mathrm{ICH}$. Second, we found that LP can not only decreased the level of high sensitive $\mathrm{C}$ reactive protein (HS-CRP), tumor necrosis factor- $\alpha$ (TNF- $\alpha$ ), NF-k $\beta$, D-dimmer (D2D), estradiol (E2), S-100B, neuron specific enolase (NSE), and interleukin 1 (IL-1) in plasma on spontaneously hypertensive rats (SHRs), but also promoted cell proliferation and inhibited cell apoptosis on the glutamate-induced PC12 cell. The compounds including Taurine, Paeonol, and Ginsenoside Rb1 in LP can activate PI3K/AKT pathway. Third, from the three-factor two-level factorial design, compound combinations in LP, such as Taurine and Paeonol, Taurine and Geniposide, Ginsenoside Rg1, and Ginsenoside Rb1, had first-level interactions on cell proliferation. Compound combinations including Taurine and Paeonol, Ginsenoside Rg1 and Ginsenoside Rb1 had as significant increase in efficiency on inhibiting the apoptosis of PC12 cells at the low concentration and up-regulating of PI3K and AKT. Overall, our results suggested that LP had integrated therapeutic effect on ICH due to activities of anti-inflammatory, anti-coagulation, blood vessel protection, and protection neuron from excitotoxicity based on the way of "multi-component, multi-target, multi-pathway," and compound combination in LP can offer protection neuron from excitotoxicity at the low concentration by activation of the PI3K/Akt signal pathway.

Keywords: intracerebral hemorrhage, liangxue tongyu prescription, network pharmacology, compound combination, PI3K/AKT 


\section{INTRODUCTION}

Intracerebral hemorrhagic (ICH) stroke is one of devastating diseases accompanied with high percentages of morbidity and mortality, causing approximately $15 \%$ of deaths from all strokes (Feigin et al., 2009; van Asch et al., 2010; Steiner et al., 2014). Brain injury following ICH is a dynamic process involving a cascade of complex pathological pathways and biochemical and metabolic events, which is approximately categorized as hemorrhagic neuroinflammation, decrease of blood flow, cytotoxic and vasogenic edema, free radical injury, rise of intracranial pressure, neuronal apoptosis and brain herniation (Lok et al., 2011; Zhou et al., 2014). These events cause recurrent strokes to make more damage to brain. However, surgical removal of the hematoma can't improve long-term prognosis dramatically, and no effective targeted therapy for hemorrhagic stroke exists yet.

Traditional Chinese Medicine (TCM) has always played a crucial role in maintaining health for over thousands of years. Indeed, prescription is the most common clinical practice of TCM, which combines several types of botanical drugs or animal medicines or minerals to fight with various diseases. Liangxue tongyu prescription (LP) is a TCM formula against stroke diseases in the clinic (Ma et al., 2011; Shi et al., 2017) including eight ingredients, namely, eight ingredients, namel Paeonia lactiflora Pall. (PR), Rheum officinale Baill. (RO), Rehmannia glutinosa (Gaertn.) Li-bosch. (RG), Panax notoginseng (Burk.)
F. H. Chen ex C. Chow. (PN), Paeonia suffruticosa Andr. (PS), Acorus tatarinowii Schott. (AT), Bubalus bubalis Linnaeus. (BB), Pheretima aspergillum (E. Perrier) (PA). Based on classical TCM theory, LP was created by Chen et al. (2016), a leading expert in the field of traditional Chinese medicine. Clinical data have proved that LP exerted its comprehensive therapeutic effects on ICH through ameliorating permeability of blood brain barrier, reducing secondary brain edema, promoting hacmatoma absorption, alleviating neuroinflammation and neurological damage (Zhang et al., 2012; Huang et al., 2014). According to the theory of TCM, LP exerts its healing effects on ICH with the effect of cooling-blood and activating-blood. Volatile oil and $n$-butanol extracts in LP were primarily identified as active fractions of LP with the effect of cooling-blood and activatingblood (Huang X. et al., 2015). However, the pharmacological effects and therapeutic efficacy of LP against ICH remain a mystery.

Based on the characteristics of complex compounds in LP, unclear targets and systemic therapy, a workflow for network-based TCM approach was proposed on ICH. The overall procedure is illustrated in Figure 1. It starts with the identification of compounds present in LP and corresponding targets on $\mathrm{ICH}$, and ends with analyzing the signaling pathways and sub-networks regulated by compounds of LP and evaluating its effects on ICH network. Further, the network prediction results were verified in vitro and in vivo experiments. On one hand, the model of spontaneously hypertensive rat (SHR)

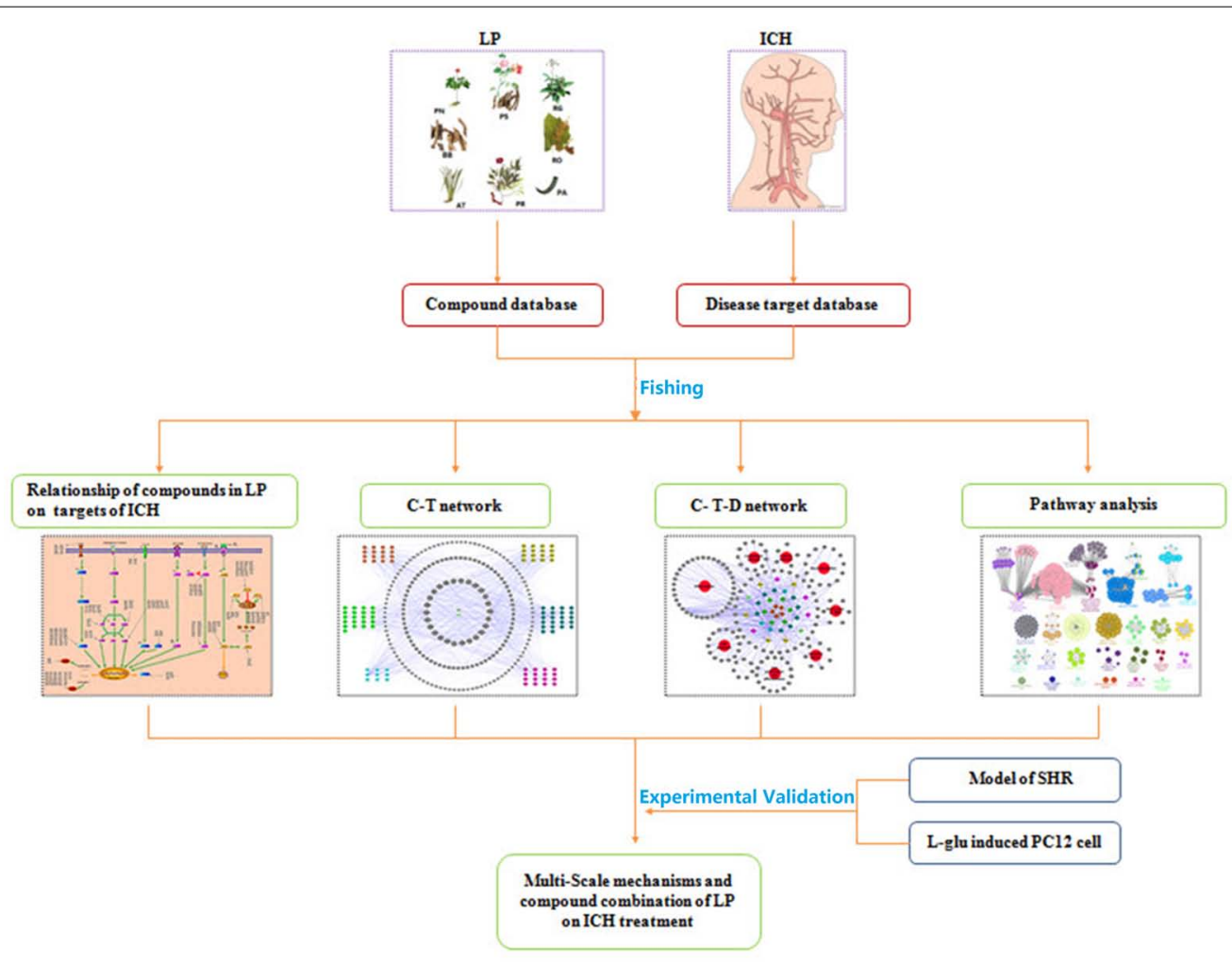

FIGURE 1 | Network pharmacology approach framework. 
and L-glutamate-damaged PC12 cells were administered to investigate the pharmacological effects of LP on ICH. On the other hand, compound combination in LP were induced to verify therapeutic efficacy of LP on the L-glutamate-damaged PC12 cells by the PI3K-AKT pathway.

\section{MATERIALS AND METHODS}

\section{ICH Associated Genes}

Multiple genes of ICH were gathered in the GeneCards ${ }^{1}$ (a database about all known diseases with a genetic component and references for further research and tools for genomic analysis of a cataloged gene), Online Mendelian Inheritance in Man database (OMIM, a database about genes, their products and biomedical applications, maintained by Israel's Weizmann Institute of Science) and related literatures with keywords of 'hemorrhagic stroke' and 'intracranial hemorrhage.' Finally, 1436 distinct targets associated with ICH diseases were dug out.

\section{All Compounds in LP}

Compounds of each ingredient in LP were collected from TCM Systems Pharmacology Database ${ }^{2}\left(\mathrm{TcmSP}^{\mathrm{TM}}\right.$, a unique systems pharmacology platform designed for herbal medicines) and pertinent literatures. Totally, 574 compounds were collected in LP. Separately, there were 120 compounds in PR, 93 compounds in RO, 77 compounds in RG, 56 compounds in PS, 120 compounds in PN, and 106 compounds in AT. However, almost no distinctive compound was found from ingredient $\mathrm{BB}$ except for taurine and PA except for lumbrokinase.

\section{The Properties of Compounds in LP}

Six significant pharmacology-related properties including number of acceptor atoms for $\mathrm{H}$-bonds (nHAcc), molecular weight (MW), number of donor atoms for H-bonds (nHDon), Moriguchi octanol-water partition coeff ( $\log \mathrm{P})$ (MLOGP), oral bioavailability (OB), and drug-likeness (DL) were acquired from $\mathrm{TcmSP}^{\mathrm{TM}}$, which reflected fundamental characteristics of each ingredient (Lipinski et al., 1997). The properties of each ingredient were analyzed based on the physicochemical properties of all compounds with $t$-test.

\section{Predicting Target Profiles of Compounds in LP}

All targets profiles associated with compounds in LP were collected from SciFinder and PubMed. Partial targets involve gene and protein files of different species, so we limited the entering species to "Homo sapiens" in the NCBI dabase ${ }^{3}$ by putting in each target that does not meet racial requirements, standardization of targets files could be got by revising to their official names. Though the transformation and retrieval, exclusive target files related with compounds in LP were finally obtained.

\footnotetext{
${ }^{1}$ http://www.genecards.org

${ }^{2}$ http://lsp.nwu.edu.cn/tcmspsearch.php

${ }^{3}$ http://www.ncbi.nlm.nih.gov/gene
}

\section{Oral Bioavailability and Drug-Likeness Screening}

The $\mathrm{OB}$ value was calculated with the in-house system OBioavail 1.1, which was applied to select out the potential compounds. In this paper, those compounds with $\mathrm{OB} \geqq 30 \%$ were chosen as potential compounds for subsequent study, and DL value of a new compound was figured by Tanimoto similarity.

$$
f(a, b)=\frac{a \cdot b}{|a|^{2}+|b|^{2}-a \cdot b}
$$

where ' $a$ ' represents the new compound, and ' $b$ ' is the average molecular properties of all compounds in Drug-Bank database (Xu et al., 2012). Through the method, compounds which satisfied the requirements with $\mathrm{OB} \geqq 30 \%$ and $\mathrm{DL} \geqq 0.1$ were chosen as potential compounds.

\section{Systems Pharmacology Network Construction and Analysis}

(1) Compound-target network (C-T network). The compounds of LP and corresponding ICH targets were applied to create C-T network with the network visualization software Cytoscape 2.8.2 (Shannon et al., 2003). (2) Potential compound-target-disease network (P-T-D network). Potential compounds of LP, targets and diseases were employed to build the P-T-D network using network visualization software Cytoscape 2.8.2. (3) Pathway analysis. 142 potential corresponding targets of potential compounds in LP were carried out pathway analysis with GlueGo to analyze biological interpretation and interrelations of functional targets in biological networks.

\section{Experimental Validation Materials}

Paeonia lactiflora Pall. (20140901), Rheum officinale Baill. (20140601), Rehmannia glutinosa(Gaertn.)Li-bosch. (20140102), Panax notoginseng (Burk.) F. H. Chen ex C. Chow. (20151021), Paeonia suffruticosa Andr. (20140901), Acorus tatarinowii Schott. (20140301), Bubalus bubalis Linnaeus (20140601), Pheretima aspergillum (E. Perrier). (20140601), were purchased from Tong Leng Hetian Chinese medicine company.

\section{Extraction Preparation}

Total extract of LP (TLP): Heat reflux extraction with water technique was applied to obtain TLP. At first, all ingredients of LP were dry matter and smashed. Then $1 \mathrm{~kg}$ mixture of LP was soaked in distilled water for $60 \mathrm{~min}$, extracted in a $20 \mathrm{~L}$ round-bottomed flask containing $10 \mathrm{~L}$ of distilled water for $1 \mathrm{~h}$ and repeated heat reflux extraction for $1 \mathrm{~h}$ with $10 \mathrm{~L}$ of new distilled water again. Then double extraction solution were combined and evaporated under reduced pressure to remove most solvents by using a rotary evaporator. Finally the extraction was dried into lyophilized powder. A total of lyophilized powder of $641.15 \mathrm{~g}$ was got with $5 \mathrm{Kg}$ mixtures of LP.

$n$-Butanol fraction extract (LPB): A total of $5 \mathrm{Kg}$ mixture of LP was extracted with heat reflux extraction with water, Then double extraction solution were combined, evaporated and extracted by equivalent volume of $n$-butanol at five times. Finally, the 
n-butanol fraction liquid was combined, evaporated and dried into lyophilized powder of $109.60 \mathrm{~g}$.

Volatile oil fraction extract (LPV): $1 \mathrm{Kg}$ mixture of LP was put in a $20 \mathrm{~L}$ round-bottomed flask containing $10 \mathrm{~L}$ of distilled water, which was connected to a Clevenger-type apparatus with tap water for cooling. The mixture of LP was under method of heat reflux extraction with water for $1 \mathrm{~h}$. Meanwhile, the obtained essential oil was collected in the side arm, then separated and dried with anhydrous sodium sulfate to eliminate moisture. Finally, the volatile oil of $9.10 \mathrm{ml}$ was obtained with $5 \mathrm{Kg}$ mixtures of LP (á $=0.91 \mathrm{~g} / \mathrm{ml})$.

\section{Durgs and Reagents}

Aspirin was purchased from by Bayer HealthCare with the batch number of J20130078). Enzyme-linked Immunosorbent Assay (ELISA) Kits including Rat interleukin 1 (IL-1), Rat S-100B protein, Rat nuclear factorkB (NF- $\mathrm{B})$, Rat neuron specific enolase (NSE), Rat high sensitive $C$ reactive protein (HS-CRP), Rat estradiol (E2), Rat tumor necrosis factor- $\alpha$ (TNF- $\alpha$ ), and Rat D-dimmer (D2D), were purchased from Nanjing Senbeijia Biological Technology Co., Ltd., Trizol was purchased from Invitrogen. Anti-AKT, Anti-PI3K, and Anti- $\beta$-actin antibody was all purchased from Abcam. All durgs (purity assay by HPLC $\geq 98 \%$, power) including compounds of Emodin, Ginsenoside Rg1, Ginsenoside Rb1, Notoginsenoside R1, Baicalein, Geniposide, $\beta$-asarone, Taurine, Paeoniflorin, Paeonol, Apigenin, Rhein, and Catalpol, were purchased from Chengdu Must Bio-Technology Co., Ltd.

\section{Animals and Prescription Administration}

48-male-(8-week-old) SHR (180-210 g), 8 male Wistar Rats (180-210 g) were purchased from Vital River Laboratory Animal Technology Co. Ltd. (License: No. SCXK (Jing) 2012-0001). All mice were housed with standard controlled conditions $(12 / 12 \mathrm{~h}$ light/dark with humidity of $40-60 \%, 21 \pm 2{ }^{\circ} \mathrm{C}$ ), and were allowed free available to food and water. Then they were acclimatized in the laboratory environment for a week prior to the experiment. The health condition of rat was checked regularly.

Eight Wistar rats as blank control groups (Distilled Water group), 48 SHR were at random assigned into six groups ( $n=8$ in each group) including model group (SHR group only), positive control group (Aspirin), LPB high dosage group, LPB low dosage group, LPV high dosage group, and LPV low dosage group. Administration of gastric infusion was executed in the dose of $10 \mathrm{~mL} / \mathrm{kg}$ once a day in each group, 15 days in total. On the 15th day, $1 \mathrm{~h}$ after administration, draw $5 \mathrm{ml}$ blood from carotid artery, and transfer it into centrifuge tube containing $0.038 \mathrm{~g} / \mathrm{ml}$ sodium citrate. Centrifuge at $3000 \mathrm{rpm}$ for $15 \mathrm{~min}$. Collect the top layer of plasma to determine indexes of NSE, IL-1, S-100B, NF- $\kappa$ B, TNF- $\alpha$, E2, D2D, and HS-CRP.

\section{Cells Culture and Treatment}

PC12 cells, kindly donated from Professor Lu (Department of Chinese Medicine Pharmacology, Nanjing University of Chinese Medicine) were cultured at $37^{\circ} \mathrm{C}$ in DMEM containing $10 \%(\mathrm{v} / \mathrm{v})$ heat-inactivated fetal bovine serum (FBS; GIBCO), $100 \mathrm{U} / \mathrm{ml}$ penicillin and $100 \mu \mathrm{g} / \mathrm{ml}$ streptomycin (Hyclone, J150019) under a humidified atmosphere of $95 \%$ air and $5 \% \mathrm{CO}_{2}$. For cell differentiation, cells were treated with $50 \mathrm{ng} / \mathrm{mL}$ of nerve growth factor (NGF; Sigma-Aldrich, USA) for 48 h. Afterwards, NGF-differentiated cultures were pretreated with different doses of each medicine at $1,5,10$, and $20 \mu \mathrm{mol} / \mathrm{L}$ for $1 \mathrm{~h}$, and then expored to $10 \mathrm{mM} L$-glutamate for an additional $24 \mathrm{~h}$. The untreated PC cells without the treatment of $L$-glutamate were used as control group.

\section{Cell Counting Kit-8 Assay}

PC12 cells were seeded into 96-well plates at a density of 50 cells $/ \mu \mathrm{L}$ and were treated by drugs in the same way as described above, and then gently washed with PBS. Afterwards, $10 \mu \mathrm{l}$ of CCK- 8 solution and $90 \mu \mathrm{l}$ of culture medium were added to each well. The 96 -well plates were maintained at $37^{\circ} \mathrm{C}$ for $4 \mathrm{~h}$ and the absorbance was measured in a microplate reader (Rayto; RT-6000) at $450 \mathrm{~nm}$. All groups were repeated in triplicate.

\section{Flow Cytometry (FCM) With Propidium lodide (PI) Staining}

The PC12 cells were seeded in 6-well plates at a density of $1 \times 10^{5} / \mathrm{ml}$ and incubated for $24 \mathrm{~h}$. Cells were treated by drugs in the same way as described above. Cells were treated with10 mM glutamate for $24 \mathrm{~h}$, then colleted and harvested with $0.25 \%$ trypsin and made into a single cell suspension, washed with cold Phosphate buffer (PBS) twice. PC12 Cells were resuspended as a single cell suspension with $50 \mu \mathrm{l}$ PBS, fixed with $1 \mathrm{ml}$ of cold $70 \%$ ethanol. Then $5 \mu \mathrm{l}$ of Annexin V-FITC and $5 \mu \mathrm{l}$ of PI stained cells in order. The cells were incubated at room temperature $\left(20-25^{\circ} \mathrm{C}\right)$ for $15 \mathrm{~min}$ in the dark and determined with a FACS Calibur flow cytometer (BectonDickinson).

\section{Real-Time Quantitative Polymerase Chain Reaction}

PC12 cells were seeded in 6 well plates and then resuspended at $1200 \mathrm{rpm}$ for $10 \mathrm{~min}$ in DMEM. Total RNA was extracted with Trizol reagent (Invitrogen, America), and the concentration of total RNA was measured with Merinton SMA4000 and the purity was estimated with the ratio of A260/A280 between 2.0 and 2.3. The first strand of cDNA was obtained with Prime Script ${ }^{\mathrm{TM}}$ RT Master Mix (Takara), and expression levels of mRNA was quantified with real-time PCR amplification instrument (Eppendorf, Germany). The program was included 1 cycle of $95^{\circ} \mathrm{C}$ for $5 \mathrm{~min}, 40$ cycles of $95^{\circ} \mathrm{C}$ for $5 \mathrm{~s}, 60^{\circ} \mathrm{C}$ for $31 \mathrm{~s}$. The mRNA levels of each gene took mRNA of GAPDH as the standard. The specific primers used in the reaction were as follows. The experiment was conducted in triplicate.

\begin{tabular}{lll}
\hline Gene & Forward primer & Reverse primer \\
\hline PI3K & GATGATITACGGCAAGATA & CACCACCTCAATAAGTCCC \\
AKT & CGGCAAGGTGATCCTGGTG & CGGTCGTGGGTCTGGAAAG \\
GAPDH & CTGGGCTACACTGAGCACC & AAGTGGTCGTTGAGGGCAATG
\end{tabular}




\section{Western Blotting}

The PC12 cells were washed with pre-cooled PBS buffer for 3 times, and lysed with RIPA buffer $(100 \mu \mathrm{l} / 50 \mathrm{ml})$ (Beyotime, Shanghai, China) and then placed for $30 \mathrm{~min}$ on ice. Cells were harvested by scraping, centrifuged at 12,000 rpm for $10 \mathrm{~min}$. Bovine serum albumin (BSA) $(2 \mu \mathrm{g} / \mu \mathrm{l})$ was diluted in PBS, and protein concentration was detected with BCA Protein Assay Kit (Pierce, Thermo Scientific) at a 50:1 ratio. The lysates $(2 \mu \mathrm{l})$ were diluted in double-distilled water $(18 \mu \mathrm{l})$ (each sample in two wells). $200 \mu l$ of detection solution were added to each well in a 96-well plates. The OD values of samples were tested at a wave length of 490 , and standard curve was drawn to calculate the concentrations of the proteins for every sample. The samples were first electrophoresed at $60 \mathrm{~V}$, and then the voltage was rised to $120 \mathrm{~V}$ as soon as the samples reached the separation gel, followed by electrophoresis (conducted at $4^{\circ} \mathrm{C}$ ) for $2 \mathrm{~h}$. After electrophoresis, the proteins were transferred onto polyvinylidene fluoride (PVDF) membranes for $2 \mathrm{~h}$ (conducted at $4^{\circ} \mathrm{C}$ ). The PVDF was removed from the transfer apparatus, and the samples were blocked with $5 \%$ evaporated milk in tris-buffered salinetween (TBST) and incubated at room temperature for $1 \mathrm{~h}$. The membranes were individually incubated with anti-phospho-Akt(1:1,000, Abcam, United Kingdom) and anti-phospho-PI3K (1:1,000, Abcam, United Kingdom) with the samples at $4^{\circ} \mathrm{C}$ overnight. After three washes with TPBS, secondary antibodies (HRP; 1:5000, Abcam, United Kingdom) corresponded to the respective primary antibodies at room temperature for $1 \mathrm{~h}$. The images were captured with the Clinx3200 + System (Clinx Science Instruments Co., Ltd., Shanghai, China), and protein densitometry was analyzed with Quantity One software (Bio-Rad, Hercules, CA, United States). Each membrane was stripped and quantified as a ratio to $\beta$-actin.

\section{Statistical Analysis}

Comparisons among multiple groups were analyzed by one-way analysis of variance (ANOVA) with post hoc test (Bonferroni or Dunnett's correction for multiple tests). For comparison between two groups, normal distribution data was analyzed by two-tailed unpaired Student's $t$-test and non-normally distributed data was analyzed by Kruskal-Wallis tests. Statistical analysis was carried out using SPSS18.0 software. $P<0.05$ or $P<0.01$ was considered as statistically significant.

\section{RESULTS}

\section{Excavation of Compounds Database of LP and Targets Database on ICH}

One thousand, four hundred and thirty-six distinct targets associated with ICH were collected from the OMIM and GenesCard database, which basically included the important disease genes of cerebral hemorrhage. Among the 1436 target genes of $\mathrm{ICH}$, inflammatory response associated genes took the lion's share with 681 targets which were $47.42 \%$ of the total disease target genes. In addition, apoptosis associated genes had577 associated genes, accouting for $40.18 \%$ of ICH targets.

A total of 572 compounds of six botanical herbs in LP were acquired from TCMSP ${ }^{\mathrm{TM}}$, Drug Bank, TTD, and related literatures, from which 120 compounds in PR, 93 in RO, 77 in RG, 56 in PS, 120 in PN, and 106 in AT were all collected. In addition, $\mathrm{PA}$ and $\mathrm{BB}$ have abundant amino acids from recent literatures (Liu et al., 2015; Yu et al., 2017).

\section{Network Analysis of Compounds in LP on ICH}

For deep understanding of the complex relationship of compounds in LP and targets of ICH from the molecular level, visual network of compounds and their corresponding targets (CT network) was constructed. At the outset, compared compounds in LP and targets of ICH, those genes that could be targeted by the corresponding compound were selected out. Then 102 compounds in LP and 200 corresponding targets were found. Second, C-T network was created with network visualization software Cytoscape. the C-T network contains 302 nodes which represents targets and compounds, and 1018 compound-target interactions (Figure 2). The mean degree value of compounds which is defined as the amount of target related to it was 9.92, indicating that most compounds exerted comprehensive effects by regulating multiple targets. Meanwhile, the external circle of targets showed lower degree value and the innermost circle of targets had the highest degree value with 22.923. It indicated that these targets may have more important positions for LP against ICH. For instance, activation of matrix metalloproteinase-9 (MMP9) can compromise blood-brain barrier, and aggravate proinflammatory gene expression and neutrophils infiltration during the formation of e perihematomal edema (Min et al., 2015). Tumor Protein P53 (TP53) gene affected mobilization of endogenous marrow-derived EPC to promote neovascularization and improve functional prognosis after ICH (Rodriguez et al., 2017). Therefore, these targets of higher value may become important therapeutic aims of LP.

Intracerebral hemorrhage is a dynamic process involving a cascade of complex pathological pathways. LP performed treating effects with its active compounds binding and regulating particular protein or nucleic acid targets. Based on the C-T network, the relationship of compounds in LP on targets of ICH was further analyzed. As shown in Figure 3, abundant compounds in LP regulated signaling pathways by adjusting and controlling these key pathway targets on ICH. Some important common pathways such as TGF- $\beta /$ smad signaling, Mitogen-Activated Protein Kinase Cascades, Apoptosis Cascades,PI3K/AKT signaling, Toll-like Receptors Pathway and NF-кB Signaling were included in the course of ICH process (Liu et al., 2016; Taylor et al., 2017; Wen et al., 2017), which covered apoptosis, proliferation, differentiation, inflammatory and immunoregulation and many other important biological processes. Meanwhile, a total of 72 compounds from eight ingredients of LP affected the prothrombotic and proinflammatory signaling pathways and regulated the AKT, MAPK, and BCL-2 pathways. These compounds may regulate the same target with coordination and antagonism or independent 


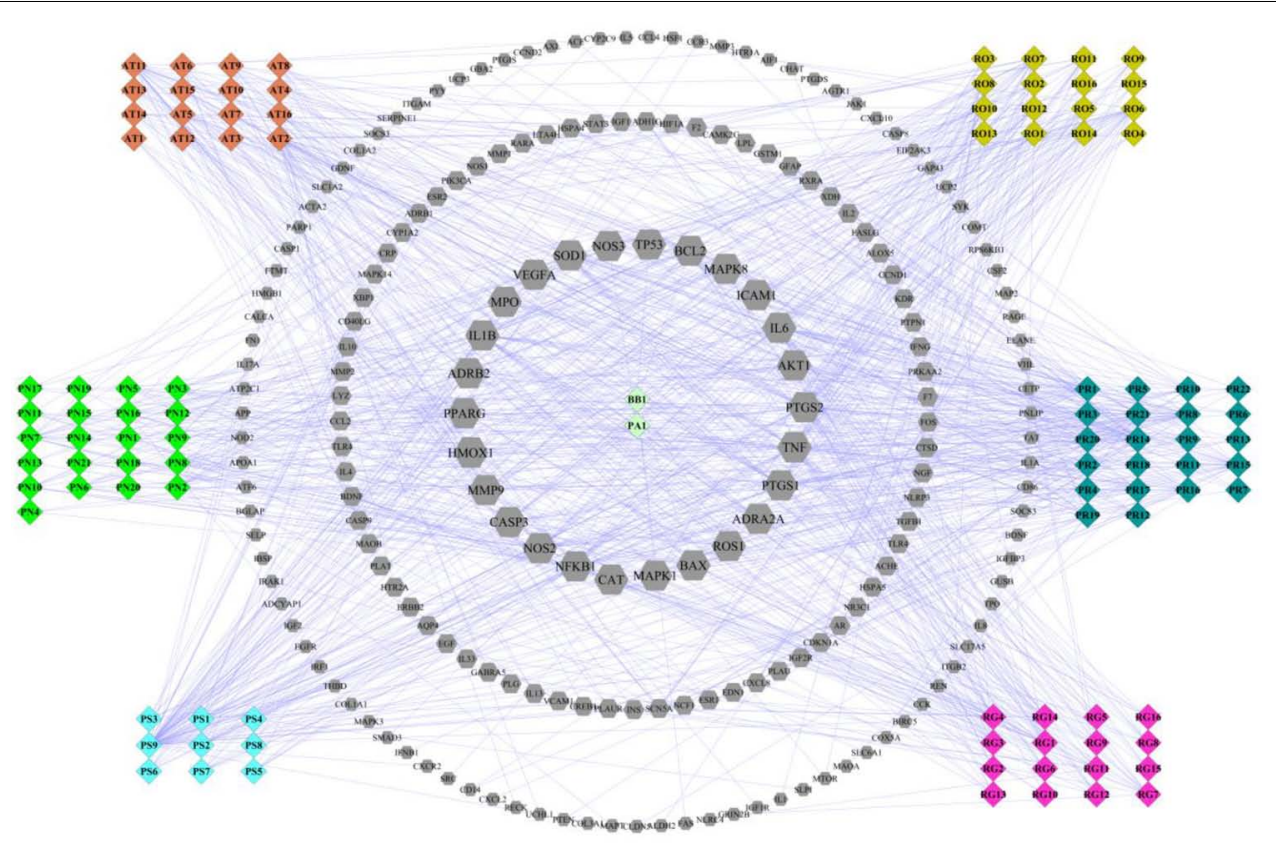

FIGURE 2 | Compound-target network of liangxue tongyu prescription (LP) on intracerebral hemorrhage. The figure was constructed by combining 102 compounds and 200 corresponding targets which were validated in published literature. Diamond represents compounds. Every color represents the single medicine. Gray hexagon represents target. Targets in the internal circle with bigger size display more relationships with compounds than these in the outside circles. PR, Paeonia lactiflora Pall.; RO, Rheum officinale Baill.; RG, Rehmannia glutinosa (Gaertn.) Li-bosch.; PN, Panax notoginseng (Burk.) F. H. Chen ex C. Chow.; PS, Paeonia suffruticosa Andr.; AT, Acorus tatarinowii Schott.; BB, Bubalus bubalis Linnaeus.; PA, Pheretima aspergillum (E. Perrier).

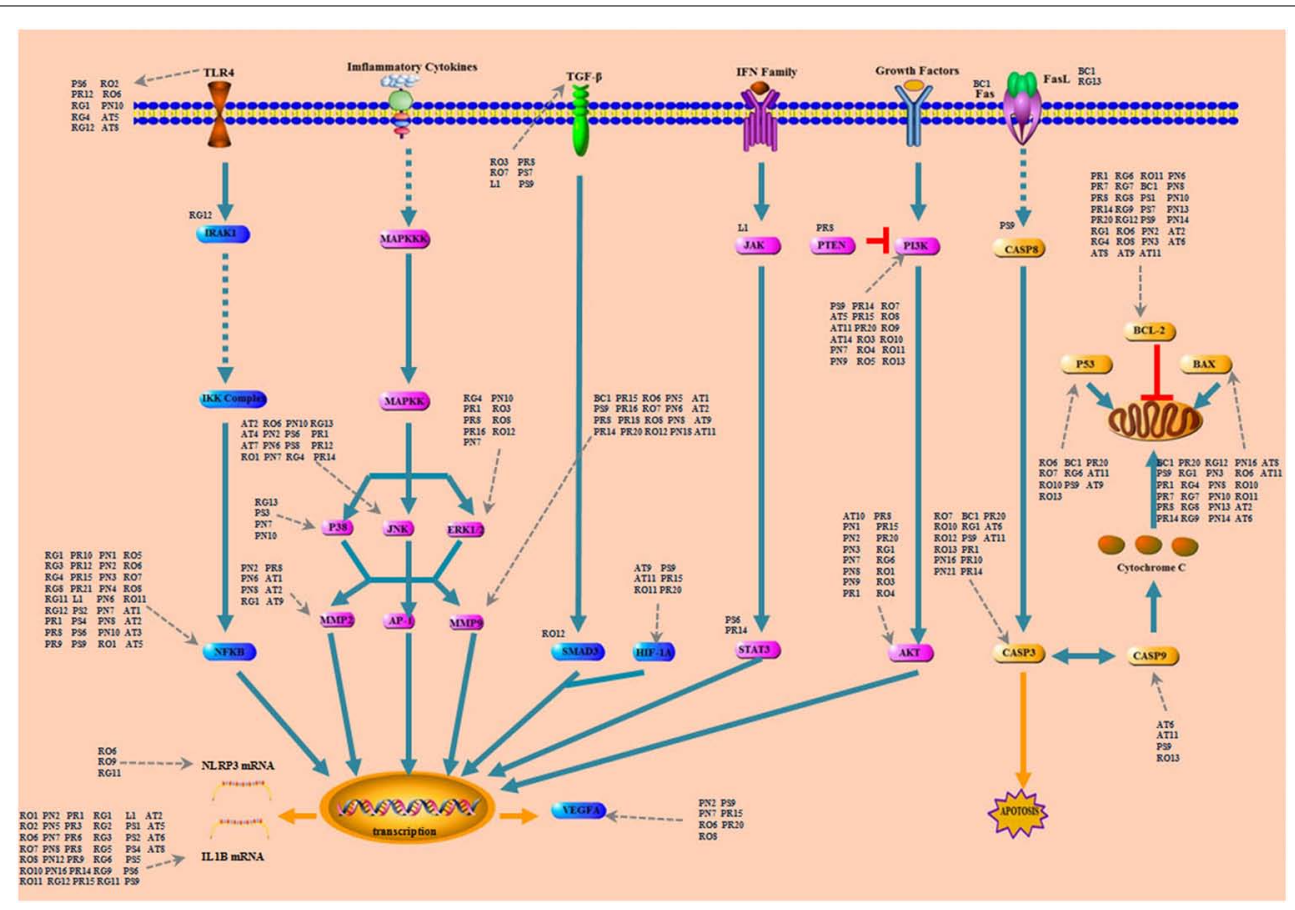

FIGURE 3 | Compounds and hitting targets on ICH. It showed an intuitional three-level structure that multi-compound hit groups of targets through multi-pathway to play the regulative role. All pathways were involved in compound control process. The nodes are pathway protein targets and the surrounding represent compounds of LP. PR, Paeonia lactiflora Pall.; RO, Rheum officinale Baill.; RG, Rehmannia glutinosa (Gaertn.) Li-bosch.; PN, Panax notoginseng (Burk.) F. H. Chen ex C. Chow.; PS, Paeonia suffruticosa Andr.; AT, Acorus tatarinowii Schott.; BB, Bubalus bubalis Linnaeus.; PA, Pheretima aspergillum (E. Perrier). 
TABLE 1 | The potential compounds in herbology of Liangxuet Tongyu Prescription (LP).

\begin{tabular}{|c|c|c|c|c|c|c|c|}
\hline NO & Compound & ОВ & DL & NO & Compound & OB & DL \\
\hline PR1 & Paeoniflorin & 53.87 & 0.79 & RG12 & $\beta$-Sitosterol & 36.91 & 0.75 \\
\hline PR3 & Ethyl linoleate & 42 & 0.19 & RG14 & Stigmasterol & 43.87 & 0.76 \\
\hline PR4 & Albiflorin & 30.25 & 0.77 & PS5 & Hederagenin & 36.91 & 0.75 \\
\hline PR8 & Paeonol & 28.79 & 0.04 & PS7 & Betulinic acid & 55.38 & 0.78 \\
\hline PR10 & Catechin & 54.83 & 0.24 & PS9 & Quercetin & 46.43 & 0.28 \\
\hline PR14 & Kaempferol & 41.88 & 0.24 & PN1 & Ginsenosides Rg1 & - & - \\
\hline PR15 & Naringenin & 33.23 & 0.24 & PN2 & Ginsenoside Rb1 & 6.24 & 0.04 \\
\hline PR19 & Oleic acid & 33.13 & 0.14 & PN3 & Notoginsenoside R1 & 5.43 & 0.13 \\
\hline PR20 & Baicalein & 33.52 & 0.21 & PN17 & (-)-alpha-Cedrene & 55.56 & 0.10 \\
\hline RO5 & Piceatannol & 72.29 & 0.13 & PN19 & Diop & 43.59 & 0.39 \\
\hline $\mathrm{RO} 7$ & Emodin & 24.4 & 0.24 & PN20 & Mandenol & 42 & 0.19 \\
\hline $\mathrm{RO8}$ & Rhein & 47.07 & 0.28 & AT2 & $\beta$-Asarone & 35.61 & 0.06 \\
\hline RO10 & Aloe-emodin & 83.38 & 0.24 & AT5 & Astragalin & 14.03 & 0.74 \\
\hline R014 & Eupatin & 50.8 & 0.41 & AT7 & N-trans-feruloyltyramine & 86.71 & 0.26 \\
\hline RG1 & Catalpol & 5.07 & 0.44 & AT11 & Apigenin & 23.06 & 0.21 \\
\hline RG6 & Geniposide & 3.78 & 0.44 & AT14 & Marmesin & 50.28 & 0.18 \\
\hline
\end{tabular}

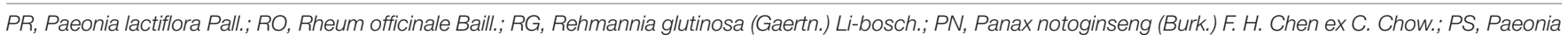
suffruticosa Andr.; AT, Acorus tatarinowii Schott. "-"represents uncollected information.

relationship. In conclusion, LP exerted its effects on ICH with multiple compounds regulating multiple pathways.

\section{Network and Pathway Analysis of Potential Compounds in LP on ICH}

\section{Potential Compounds in LP Screening}

With the purpose of screening out the potential compounds in LP, OB and DL evaluation were applied. According to screening rules of $\mathrm{OB} \geqq 30 \%$ and $\mathrm{DL} \geqq 0.1$, a total of 32 potential compounds of six herbal medicines in LP were selected out (Table 1). However, some compounds among them which couldn't meet the requirements, were confirmed by experimental evidence and then were also allocated to potential compounds. For example, Catalpol has been displayed that it had the properties of anti-apoptosis, anti-inflammation and neuroprotection against Alzheimer's disease, ischemic stroke and idiopathic Parkinson's disease (Jiang et al., 2015). Geniposide enhanced growth factor signaling and inhibited apoptosis of neurone in the mouse model of Parkinson disease to exert neuroprotective effect (Jiang et al., 2015). $\beta$-Asarone was also added as potential compound for further analysis because $\beta$-Asarone treatment could suppress Beclin-1-dependent autophagy through the PI3K/Akt/mTOR signal pathway (Deng et al., 2016). Panax notoginseng saponins (PNS) were mainly composed of Ginsenoside Rb1, Ginsenoside Rg1, and Notoginsenoside R1, which had positive protection on ischemia brain damage with the activity of anti-inflammation and anti-apoptosis (Huang X.P. et al., 2015). Paeonol may possess anti-inflammatory and anti-oxidant functions with its active metabolites (Jin et al., 2016). In addition, Taurine was found that it promoted synapse development and proliferation of neuron (Liu et al., 2010). Lumbrokinase may relieve ischemia-reperfusion injury via TLR4 signaling (Wang et al., 2016). In all, It was found that 34 potential compounds in LP were preserved for subsequent analysis.

\section{Network Analysis of Potential Compounds in LTF on ICH}

Traditional Chinese Medicine which involved many bioactive compounds may hit one or multiple targets from the network of biology. Then the biological system achieved new balance to reduce the harmful effects. Typically, Potential compound-targets- disease (P-T-D) network in LP on $\mathrm{ICH}$ was constructed to forecast and interpret the poly pharmacology action of multi-compound and multi-target. As shown in Figure 4, there are 34 potential compounds and 146 corresponding targets with the connections also reaching 506 items which indicates the close relationships between them. Lots of associated targets independently belong to inflammation (36/146), cell apoptosis (21/146), cell proliferation and differentiation (22/146), oxidative stress (7/146), destruction of blood-brain barrier (7/146), neurotransmitter (13/146), calcium overload(5/146), and coagulation cascade (11/146), which implied that the compounds of LP may intervene many pathologies of ICH such as neurologic function, thrombosis, oxidative stress, cell apoptosis, and inflammation. For example, prothrombin (F2) and coagulation factor VII (F7) are important coagulation factors in the thrombosis process. The injured vessels induce these circulating coagulation factors in a chain of interlacing reactions, causing development of a coagulum to generate thrombosis, and multicompound in LP might regulate coagulation factors to put into effect.

\section{Pathway Analysis of Targets Associated With Potential Compounds in LP on ICH}

A total of 142 corresponding targets rooted in P-T-D network were chosen as potential ICH targets to carry on Go analysis. As shown in Figure 5, 24 signaling pathways of ICH associated with potential compounds in LP were fully excavated by 


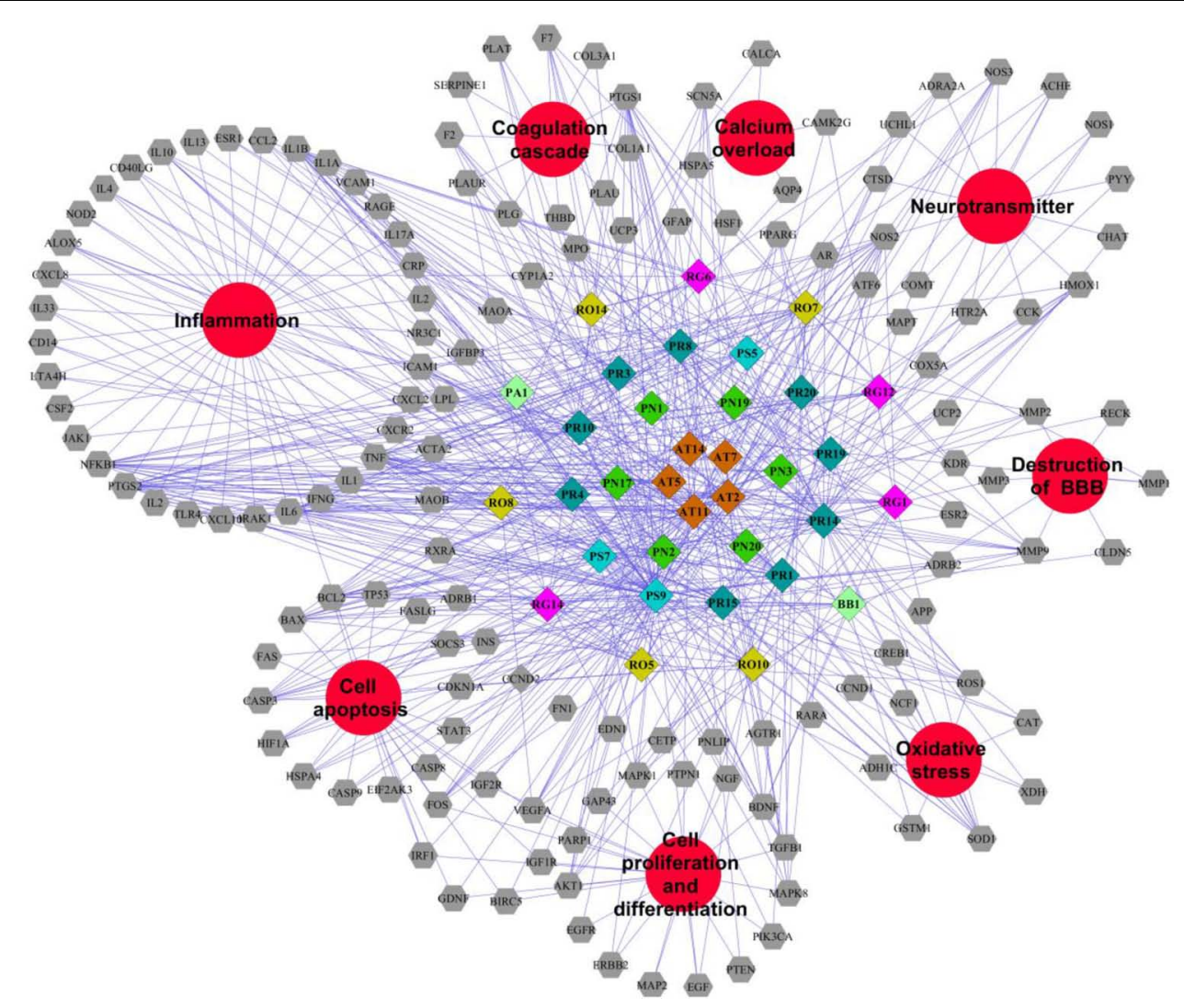

FIGURE 4 | Potential compound-targets-disease network in LP on ICH. There were 34 potential compounds, 146 corresponding targets and eight pathologyies types on ICH in the P-T-D network. Diamond represents potential compounds, each color represents one herb in LP respectively; Gray hexagon represents targets while the red circles represent the pathologyies types of ICH. PR, Paeonia lactiflora Pall.; RO, Rheum officinale Baill.; RG, Rehmannia glutinosa (Gaertn.) Li-bosch.; PN, Panax notoginseng (Burk.) F. H. Chen ex C. Chow.; PS, Paeonia suffruticosa Andr.; AT, Acorus tatarinowii Schott.; BB, Bubalus bubalis Linnaeus.; PA, Pheretima aspergillum (E. Perrier).
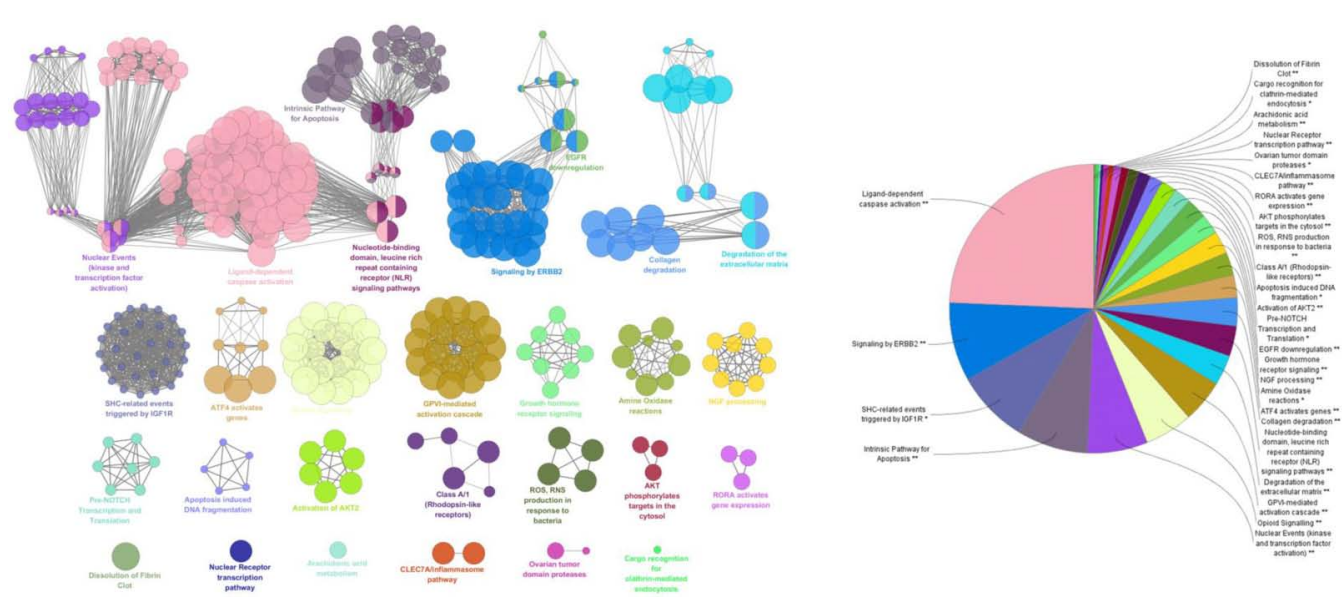

FIGURE 5 | ClueGO founctional analysis of the potential targets. GO terms and pathways representative for target genes are selected and visualized with a functionally grouped network, the GO terms render as nodes, and the significance is represented with node size. Functionally related groups are able to partially overlap. The node pie charts stand for the reactome analysis of targets. Only the most important term was labeled. The above GlueGO setting was representative reactome analysis among potential targets. 
REACTOME pathways with 9841 available unique genes. The main pathways were involved in inflammation, coagulation, apoptosis and other pathologic links and relevant signaling pathways. Specifically, the reactome pathways were mainly related to Ligand-dependent caspase activation, signaling by ERBB2, SHC-related events triggered by IGF1R and intrinsic pathway for Apoptosis. Moreover, although some pathways didn't occupy larger proportion, they also display tremendous importance, such as dissolution of Fibrin Clot, inflammasome pathway, AKT phosphorylates targets in the cytosol, and degradation of the extracellular matrix. These reactome pathways have been linked to the internal mechanism of hemorrhagic stroke and indicated the complexity of disease pathological process in more depth. Protein targets of ICH were shown to be involved in autophagy, ischemia, necrosis, apoptosis, calpain activation, inflammation, oxidative stress, caspase activation and cytokine secretion based on the current level of global pathway analysis. Go analysis resulted that these potential protein targets of potential compounds in LP were involved in specific pathological pathways basically.

The perihematomal region resulted in a prominent form of cell death during the development of ICH. Therefore, inhibition of the neuron apoptosis has been expected to improve functional recovery and reduce tissue damage in ICH. Among the pathways of ICH associated with potential compounds in LP, the PI3K-AKT pathway wasn't dominant position, but it had obvious upstream and downstream proteins which played a significant role in neuronal apoptosis, and an important contributor to early brain injury after ICH by taking part in cell survival and proliferation and regulating some important caspase proteins and apoptotic genes. As shown in Figure 6, PI3K stimulated the generation of the lipid second messenger, converting phosphatidylinositol $(4,5)$ bisphosphate (PIP2) into phosphatidylinositol $(3,4,5)$ trisphosphate (PIP3). PTEN antagonized action of PI3K, and Akt resorted its pleckstrin homology domain to bind the $3^{\prime}$-phosphorylated inositol lipids. Compounds in LP have interferential effect on the phosphorylation of PI3K and AKT proteins based on the dissection of network pharmacology. PI3K may be targeted by 19 compounds from eight ingredients of LP such as Emodin, Baicalein, Ginsenoside Rg1, and so on. AKT may be targeted by 17 compounds from eight ingredients of LP such as Catalpol, Paeoniflorin, Paeonol, Ginsenoside Rg1, Ginsenoside Rb1, and so on. PI3K inhibitor could be targeted by only one compound Paeonol. The PI3K-Akt pathway acted an important part in inhibition of apoptosis and neuroprotection (Wang et al., 2013). The downsteam target AKT influences many other pathways such as inhibition of caspase and the regulation of Bcl-2 family proteins, NF-k $\beta$ pathway, JNK pathway, and ERK pathway (Jacquin et al., 2013; McNamara et al., 2013; Zhou et al., 2015; Velmurugan et al., 2017).

\section{Experimental Verification on ICH} Pharmacodynamic Evaluation of LP on SHR Model

Based on the network pharmacological prediction, the compounds of LP may act on different pathologies of ICH

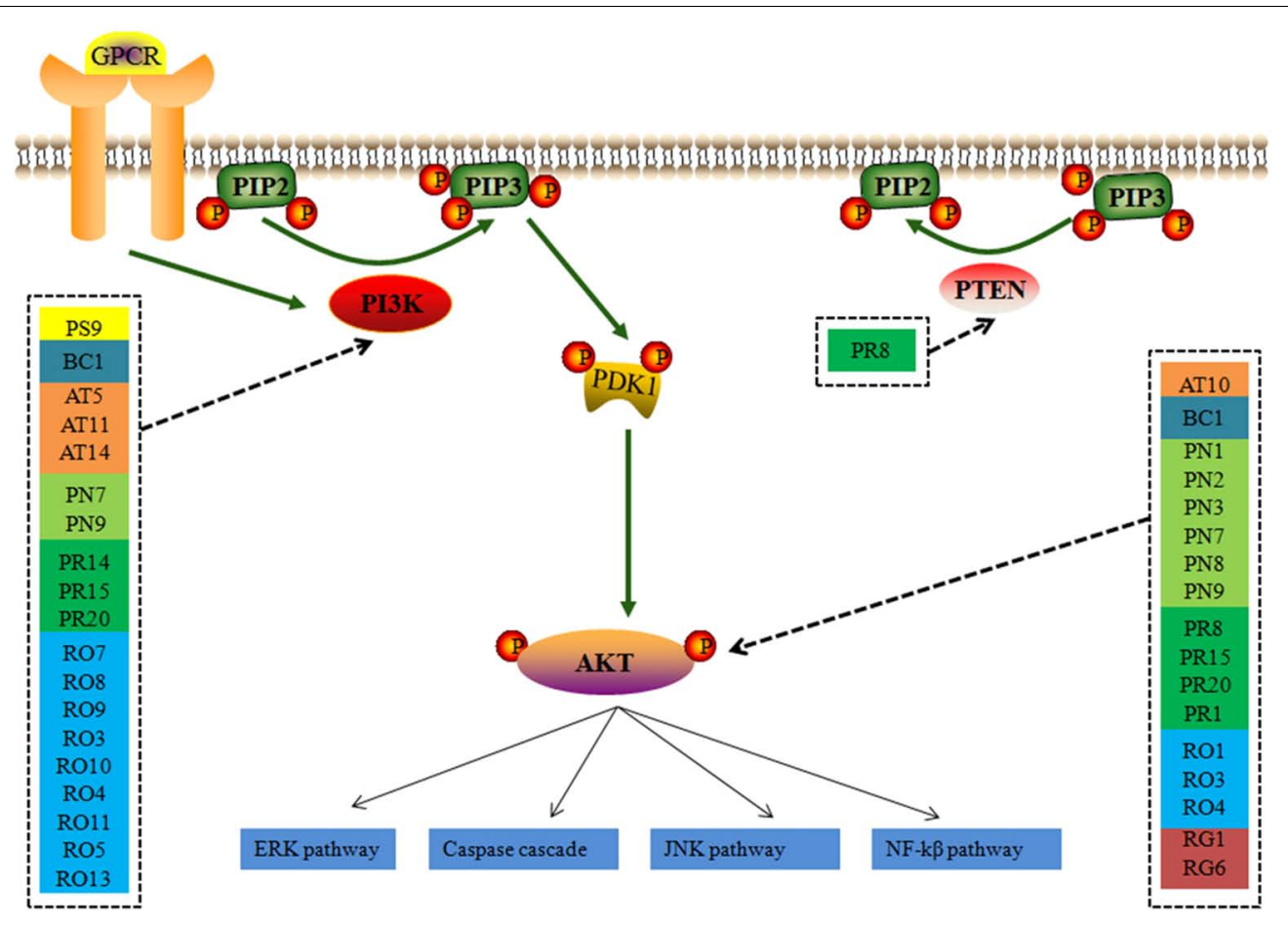

FIGURE 6 | The simplified PI3K-AKT pathway regulation of compounds in LP. Radix Paeoniae Rubra. RO, Rheum officinale Baill.; RG, Rehmannia glutinosa (Gaertn.) Li-bosch.; PN, Panax notoginseng (Burk.) F. H. Chen.; PS, Paeonia suffruticosa Andr.; AT, Acorus tatarinowii Schott.; BB, Bubalus bubalis Linnaeus.; PA, Pheretima aspergillum (E. Perrier). 

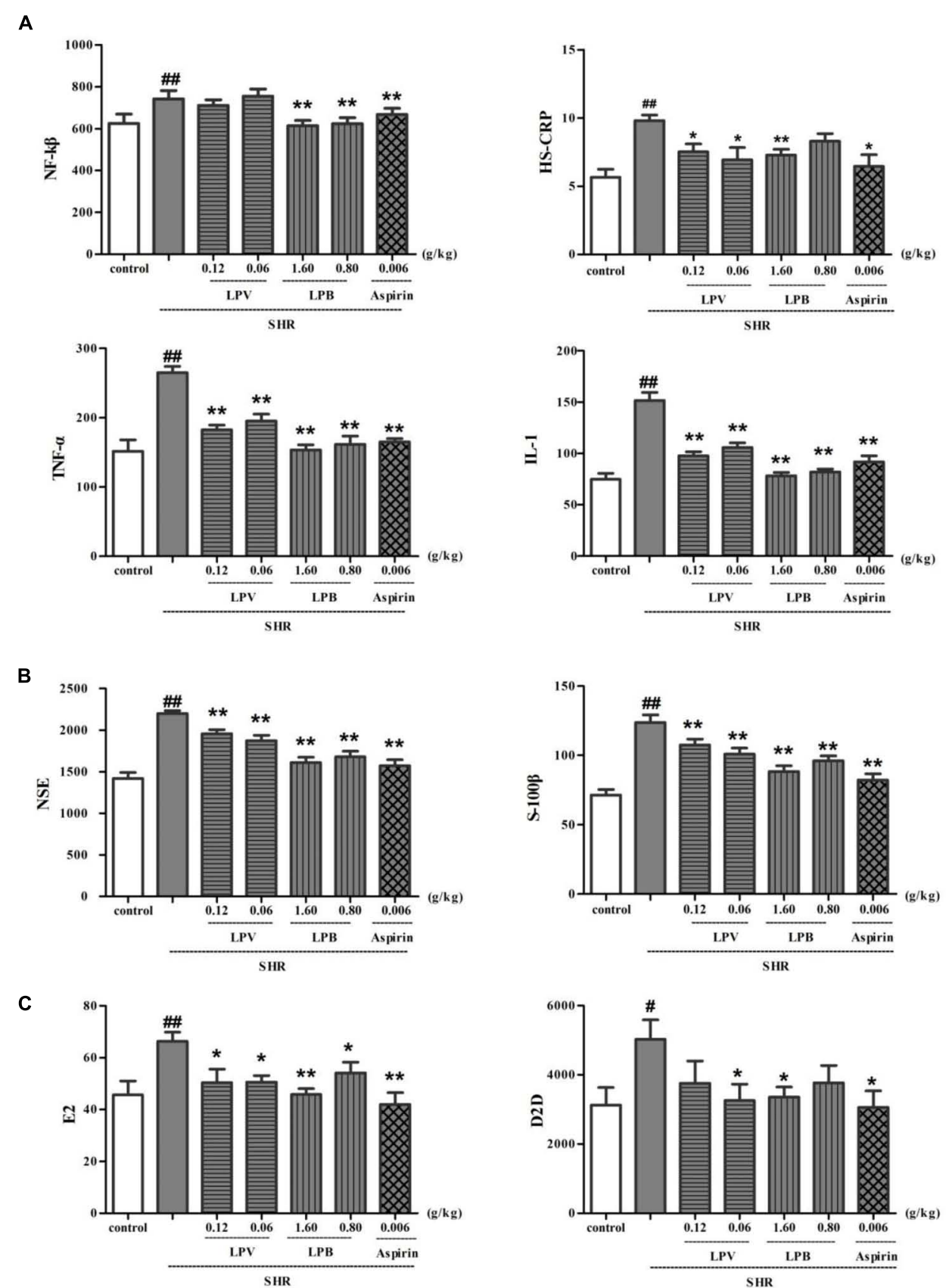

FIGURE 7 | Effects of LPB and LPV from LP and aspirin on levels of IL-1, NF-KB, TNF- $\alpha$, HS-CRP (A), NSE, S-100B (B), D2D and E2 (C) in serum on SHR model. Data were expressed as the mean \pm SEM for six rat $\left({ }^{*} P<0.05,{ }^{* *} P<0.01\right.$, vs. SHR group only (model group); ${ }^{*} P<0.05$, \#\# $P<0.01$, vs. Control). LP, Liangxue tongyu prescription; LPV, Volatile oil fraction extract of LP; LPB, $n$-butanol fraction extracts of LP; Glu, glutamate; SHR, spontaneously hypertensive rat.

such as neurologic function, thrombosis and inflammation. $\mathrm{ICH}$ stands for the acute presentation of cerebral small-vessel disease. Persistently raised intraluminal arterial pressure destories small-vessel walls and hypertension becomes the leading cause of ICH (Biffi et al., 2015). The level of IL-1, HS-CRP, NF- $\kappa$ B, S-100B protein, TNF- $\alpha$, NSE, D2D, and E2 were detected in SHR treated with extracted fractions from LP. The LPV and LPB, two effective extracted fractions in LP for anti-inflammatory and activating blood on blood-heat and blood-stasis rat model, were adopted to estimate pharmacodynamic evaluation of LP on SHR model.

High sensitive $C$ reactive protein, TNF- $\alpha, N F-k \beta$, and IL-1 levels in plasma in model group were obviously higher than that in control group $(P<0.01$, Figure 7A). HS-CRP, TNF- $\alpha$, NF-k $\beta$, and IL-1 levels in aspirin group as positive medicine (Chong et al., 2012) was observably lower than that in model group $(P<0.01$, 
TABLE 2 | Effects of extract of $L P$ and its extracted fractions including LPB, LPV, and TLP on the cell proliferation in L-Glu-damaged PC12 cell.

\begin{tabular}{lcc}
\hline Group & Dose $(\mu \mathbf{g} / \mathbf{m L})$ & OD450 \\
\hline Control & - & $1.292 \pm 0.081$ \\
Glu & - & $0.891 \pm 0.054^{\# \#}$ \\
Glu + TLP & 64.118 & $1.166 \pm 0.120^{* *}$ \\
Glu + TLP & 128.235 & $1.208 \pm 0.057^{* *}$ \\
Glu + TLP & 256.469 & $1.300 \pm 0.063^{* *}$ \\
Glu + TLP & 512.938 & $1.302 \pm 0.052^{* *}$ \\
Glu + LPB & 10.959 & $1.640 \pm 0.037^{* *}$ \\
Glu + LPB & 21.917 & $1.208 \pm 0.043^{* *}$ \\
Glu + LPB & 43.833 & $1.307 \pm 0.038^{* *}$ \\
Glu + LPB & 87.665 & $1.333 \pm 0.065^{* *}$ \\
Glu + LPV & 0.818 & $0.944 \pm 0.012$ \\
Glu + LPV & 1.636 & $0.967 \pm 0.021$ \\
Glu + LPV & 3.723 & $1.018 \pm 0.023$ \\
Glu + LPV & 6.545 & $1.125 \pm 0.051^{* *}$ \\
\hline
\end{tabular}

Data are expressed with Mean \pm SEM. ${ }^{\#} P<0.05$, \# $P<0.01$, vs. control. ${ }^{*} P<0.05,{ }^{*} P<0.01$, vs. model (only Glu-damaged group). Each extract has four doses corresponding to the dose of crude medicine at 4000, 2000, 1000, and $500 \mu \mathrm{g} / \mathrm{ml}$. Glu:glutamate, LPV, Volatile oil fraction extract of LP; $L P B, n$-butanol fraction extracts of $L P ; T L P$, total extract of $L P ; L P$, liangxue tongyu prescription.

$P<0.05)$. Compared to model group, after treatment with different doses of LPB and LPV, HS-CRP, TNF- $\alpha$ and IL-1 levels in serum were all significantly decreased $(P<0.05)$ and NF-k $\beta$ levels in LPB treatment groups were all significantly decreased $(P<0.01)$.

S-100B and NSE level in model groups increased remarkably in contrast to control group $(P<0.01$, Figure $7 \mathbf{B})$. In comparison with model group, level of S-100B and NSE in aspirin, LPV and LPB groups decreased significantly $(P<0.01)$. Compared with control group, D2D and E2 level in model group show a significant difference $(P<0.01$, Figure 7C). Compared with model group, D2D and E2 level of aspirin, LPV and LPB groups reduced significantly $(P<0.05, P<0.01)$. The $\mathrm{D} 2 \mathrm{D}$ is very sensitive to intravascular thrombus and may be markedly elevated in disseminated intravascular coagulation. E2 exerted a protective effect by intracellular $\mathrm{Ca}^{2+}$ levels (Koh, 2014).

\section{Effects of Extracted Fractions and Compounds of LP on L-Glutamate-Induced PC12 Cell Proliferation}

Cell proliferation was evaluated on the glutamate-induced PC12 cell treated with extracted fractions and compounds of LP. As is shown in Table 2, L-glutamate obviously inhibited cell proliferation in comparison to control group $(p<0.01)$, different dosages of LPB and TLP all can enhanced proliferation of PC12 cells $(p<0.01)$. The group dealt with LBV only enhanced cell viability at $6.435 \mu \mathrm{g} / \mathrm{mL}(p<0.01)$.

In the meanwhile, Emodin, GRb1, Catalpol, Paeonol, GRg1, NR1, Baicalein, Geniposide, Taurine, $\beta$-asarone could effectively increase proliferation of PC12 cells at $20 \mu \mathrm{M}(p<0.05, p<0.01$, Figure 8). GRb1, Geniposide and Taurine showed fine effects with the lowest concentration of $5 \mu \mathrm{M}(p<0.01)$. Therefore, these compounds except Rhein, Paeoniflorin and Apigenin all increased cell proliferation on $L$-glutamate-induced PC12 cell.

\section{Effects of Extract of LP and Its Extracted Fractions on L-Glutamate-Induced PC12 Cell Apoptosis}

To ascertain the anti-apoptotic effect of LP, apoptosis rate of pretreatment with TLP, LPB, and LPV were evaluated on L-glutamate-induced PC12 cell. $10 \mathrm{mM}$ glutamate was able to induce PC12 cells apoptosis from 3.79 to $19.03 \%$ in $24 \mathrm{~h}(P<0.01$, Figure 9A). However, pretreatment with TLP, LPB, or LPV for $1 \mathrm{~h}$ prior to glutamate exposure decreased the apoptosis rate to $5.39 \%$ (LPB), 4.06\% (TLP), and 5.65\% (LPV), respectively $(P<0.01)$. Therefore, LP had obvious neuroprotective effect when PC12 cells were exposed to amino acid damage.

\section{Effect of Compound Combination in LP Properties Analysis of Total Compounds in LP}

For determining whether the six herbal ingredients in LP were different or similar on compound composition, total compounds in six herbs were compared under important properties including MW, nHAcc, nHDon, OB, MlogP, and DL. On one hand, the distribution interval of various properties for all compounds in six medicinal herbs of LP show obvious difference. On the other hand, properties of total compounds of blood-activating herbs such as PS and PN undifferentiated with PR in MW, nHDon, nHAcc, MlogP, and OB, properties of total compounds of heat-clearing herb such as RO only had subtle differences in MW and MlogP compared to RG ( $p<0.05$, Table 3 ). However, six properties of total compounds in AT for resuscitation and palinesthesia of consciousness almost showed significant differences with other herbs $(P<0.01)$. The results showed that although there were various compounds in the various herbs of LP, the properties of heat-clearing herbs as PR and PS were similar, the same as RO and RG as blood-activating herbs.

\section{Effects of Compound Combination in LP on L-Glutamate-Induced PC12 Cell Proliferation}

According to the prediction result of PI3K/AKT pathway on $\mathrm{ICH}$ in LP (Figure 2), effect of compound combination in LP on L-glutamate-induced PC12 cell proliferation was further investigated with the three-factor two-level factorial design. The result showed that first-level interactions existed between Taurine and Paeonol, Taurine and Geniposide, Paeonol and Geniposide, Ginsenoside Rg1 and Ginsenoside Rb1 $(p<0.01$, Table 4), but secondary interaction didn't exist among compound combination. Taurine was a characteristic compound from BB and Geniposide was an important compound from RG. BB and RG were classic herb pair in TCM, therefore compound combination of taurine and geniposide was reserved for further study. Potential compounds Ginsenoside Rg1 and Ginsenoside $\mathrm{Rb} 1$ were from the same herb of $\mathrm{PN}$, which also were reserved to explore the effect of compound combination by PI3K/AKT pathway.

\section{Effects of Compound Combination on L-Glutamate-Induced PC12 Cell Apoptosis}

Based on the effect of the compound combination of LP in the experiment above (Table 4), combination of Ginsenoside Rg1 and Ginsenoside Rb1, Taurine and Geniposide on neuronal 

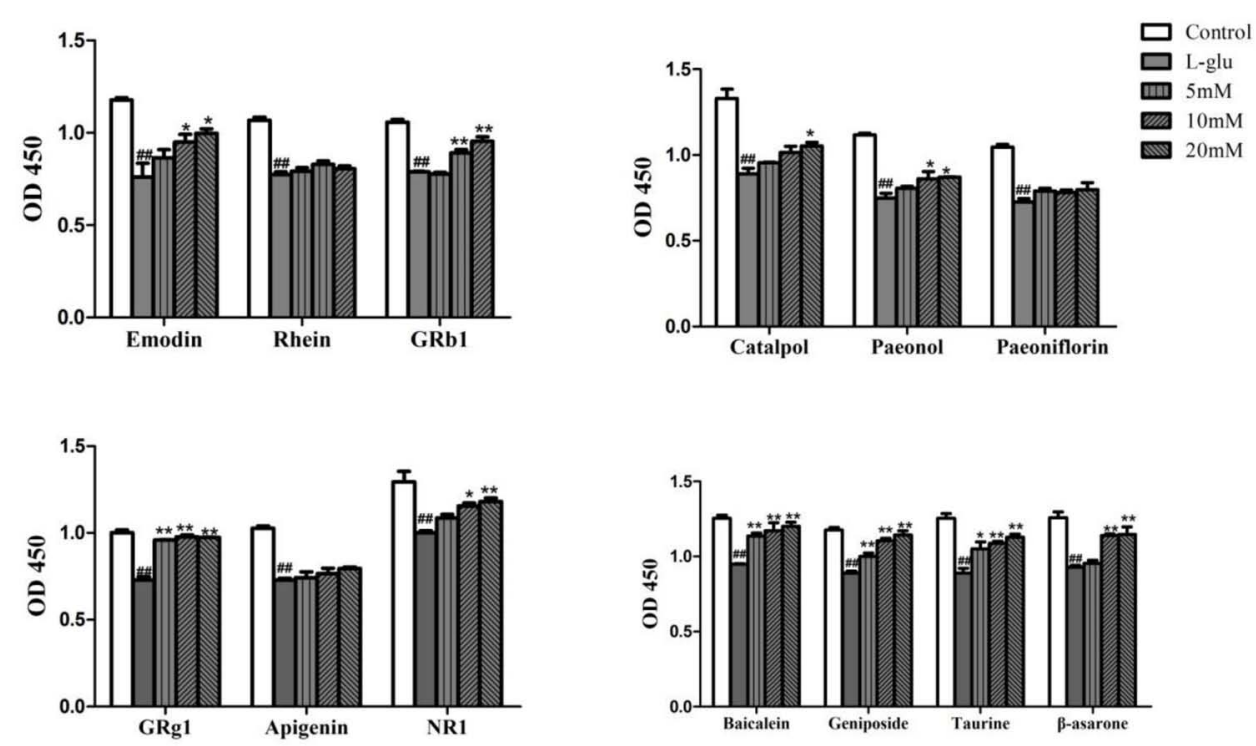

FIGURE 8 | Effects of compounds in LP on the cell proliferation on L-Glu-damaged PC12 cell. Data are expressed as mean \pm SEM. \# $\mathrm{P}<0.05$, \#\# $\mathrm{P}<0.01$, vs. control. ${ }^{*} P<0.05,{ }^{*} P<0.01$, vs. model (only Glu-damaged group). Glu, glutamate; GRg1, ginsenoside rg1; GRb1, ginsenoside rb1; NR1, Notoginsenoside R1.

A

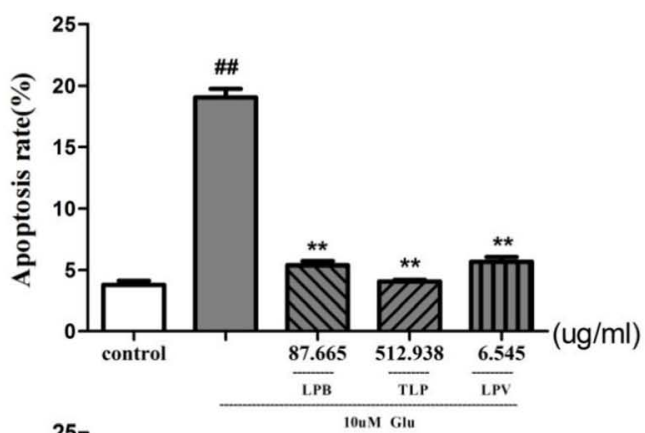

B
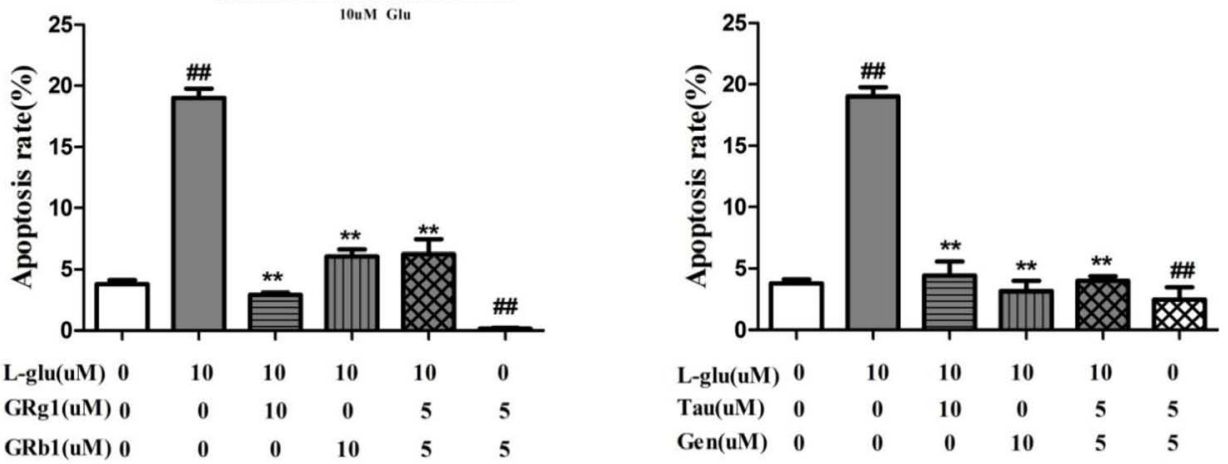

FIGURE 9 | Effect of LP on apoptosis of PC12 cell induced by L-glutamate. (A) Extract of LP and its extracted fractions. Each group of extract has the highest dose corresponding to the dose of crude medicine at $4000 \mu \mathrm{g} / \mathrm{ml}$. (B) Comounds of LP including Taurine, Geniposide, GRg1, and GRb1. Data are shown as the means \pm SEM from three replicate experiments. ${ }^{*} P<0.05,{ }^{* *} P<0.01$, vs. L-Glu; ${ }^{\#} P<0.05$, ${ }^{\# \#} P<0.01$, vs. control. LPV, volatile oil fraction extract of LP; LPB, n-butanol fraction extracts of LP; TLP, total extracts of LP; L-Glu, L-glutamate; GRg1, Ginsenoside Rg1; GRb1, Ginsenoside Rb1; Tau, Taurine; Gen, Geniposide.

apoptosis were evaluated with the model of $L$-glutamate-induced cell apoptosis. The apoptosis radio of cells was increased from 3.79 to $19.03 \%$ after the cells were treated with $10 \mathrm{mM}$ glutamate for $24 \mathrm{~h}(p<0.01$, Figure 9B). The combination of Taurine and Geniposide, Ginsenoside Rgland Rb1 at $5 \mu \mathrm{M}$ effectively decreased percentage of apoptosis on $L$-glutamateinduced PC12 cell and normal PC12 cell obviously $(p<0.01)$, and were the same as at $10 \mu \mathrm{M}$ respectively $(P<0.01)$. The 


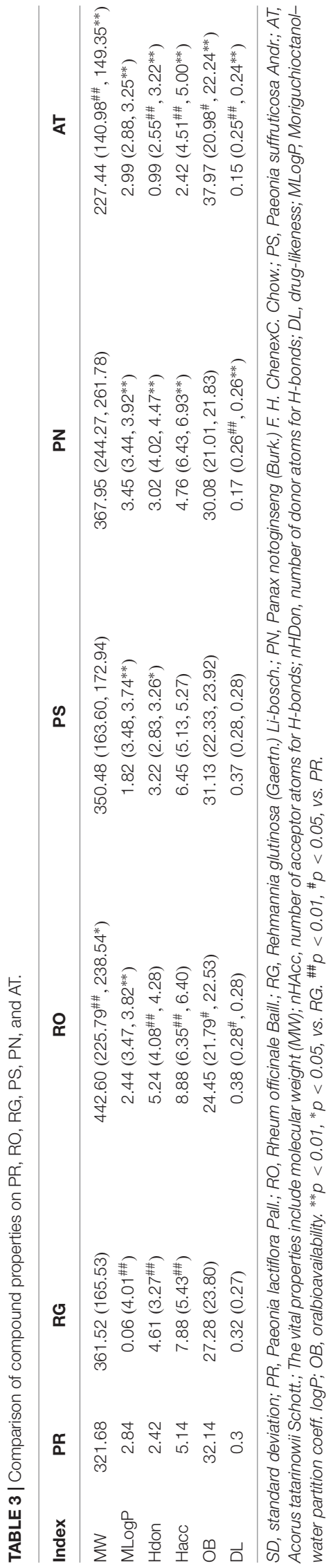

TABLE 4 | Results of compound combination in LP on L-glutamate-induced PC12 cell proliferation with factorial analysis of three-factor two-level design.

\begin{tabular}{|c|c|c|c|c|}
\hline \multirow{2}{*}{$\begin{array}{l}\text { Tset } \\
\text { order }\end{array}$} & \multicolumn{3}{|c|}{ Dosage level ( $\mu \mathrm{mol} / \mathrm{L})$} & \multirow[t]{2}{*}{ OD450 $(n=5)$} \\
\hline & Taurine & Paeonol & Geniposide & \\
\hline 1 & 2.5 & 2.5 & 2.5 & $1.526 \pm 0.043$ \\
\hline 2 & 2.5 & 2.5 & 5.0 & $1.513 \pm 0.087$ \\
\hline 3 & 2.5 & 5.0 & 2.5 & $1.502 \pm 0.059$ \\
\hline 4 & 2.5 & 5.0 & 5.0 & $1.518 \pm 0.029$ \\
\hline 5 & 5.0 & 2.5 & 2.5 & $1.517 \pm 0.078$ \\
\hline 6 & 5.0 & 2.5 & 5.0 & $1.314 \pm 0.087$ \\
\hline 7 & 5.0 & 5.0 & 2.5 & $1.285 \pm 0.048$ \\
\hline 8 & 5.0 & 5.0 & 5.0 & $1.261 \pm 0.087$ \\
\hline \multirow{2}{*}{$\begin{array}{l}\text { Tset } \\
\text { order }\end{array}$} & \multicolumn{3}{|c|}{ Dosage level $(\mu \mathrm{mol} / \mathrm{L})$} & OD450 $(n=6)$ \\
\hline & $\begin{array}{l}\text { Ginsenoside } \\
\quad \text { Rg1 }\end{array}$ & $\begin{array}{c}\text { Ginsenoside } \\
\text { Rb1 }\end{array}$ & $\begin{array}{l}\text { Notoginsenoside } \\
\text { R1 }\end{array}$ & \\
\hline 1 & 2.5 & 2.5 & 2.5 & $1.645 \pm 0.053$ \\
\hline 2 & 2.5 & 2.5 & 5.0 & $1.626 \pm 0.036$ \\
\hline 3 & 2.5 & 5.0 & 2.5 & $1.643 \pm 0.034$ \\
\hline 4 & 2.5 & 5.0 & 5.0 & $1.578 \pm 0.053$ \\
\hline 5 & 5.0 & 2.5 & 2.5 & $1.533 \pm 0.070$ \\
\hline 6 & 5.0 & 2.5 & 5.0 & $1.487 \pm 0.055$ \\
\hline 7 & 5.0 & 5.0 & 2.5 & $1.380 \pm 0.061$ \\
\hline 8 & 5.0 & 5.0 & 5.0 & $1.283 \pm 0.045$ \\
\hline
\end{tabular}

Tests of Between-Subjects Effects: Taurine vs. Paeonol, $p<0.01$; Paeonol vs. Geniposide, $p<0.01$; Taurine vs. Geniposide, $p<0.01$; Ginsenoside $R g 1 \times$ Ginsenoside Rb1, $p<0.01$. Cells were treated with combined compounds at two kinds of concentration (2.5 and $5 \mu \mathrm{mol} / \mathrm{L}) 1 \mathrm{~h}$ before and glutamate $(10 \mathrm{mM})$ for additional $24 \mathrm{~h}$. Data are expressed from five replicate experiments as the means \pm SEM.

results showed that the combination of Ginsenoside Rg1 and $\mathrm{Rb} 1$, taurine and geniposide in LP at low concentration had a significant increase in efficiency on inhibition of neuron apoptosis.

\section{Effects of Compounds and Their Combination in LP on PI3K/AKT Pathway}

In order to verify regulation effect of compounds and their combination in LP on PI3K/AKT signaling pathway, the mRNA and protein levels of PI3K and AKT were measured with RT-QPCR and western blot (Figure 10). Compared with normal groups, $L$-Glu-damaged groups showed down-regulated of PI3K and AKT mRNA and protein expression $(P<0.01)$. Compared with $L$-Glu-damaged groups, GRb1 upregulated PI3K and AKT mRNA expression $(P<0.01)$. The combination of taurine and geniposide at $5 \mu \mathrm{M}$ upregulated AKT mRNA the expression $(P<0.05)$. However, Taurine or Geniposide at $10 \mu \mathrm{M}$, and combination of Taurine and Geniposide at $5 \mu \mathrm{M}$ up-regulated $\mathrm{p}$-AKT and $\mathrm{p}$-PI3K protein expression on $L$-Glu-damaged PC12 cell $(P<0.01)$. GRb1 at $10 \mu \mathrm{M}$, and combination of GRb1and GRg1 at $5 \mu \mathrm{M}$ also up-regulated p-AKT and p-PI3K protein expression on $L$-Glu-damaged PC12 cell $(P<0.01)$. In addition, combination of GRbland GRg1 at $5 \mu \mathrm{M}$ up-regulated $\mathrm{p}$-AKT and $\mathrm{p}$-PI3K protein expression on normal PC12, while combination of Taurine 
A
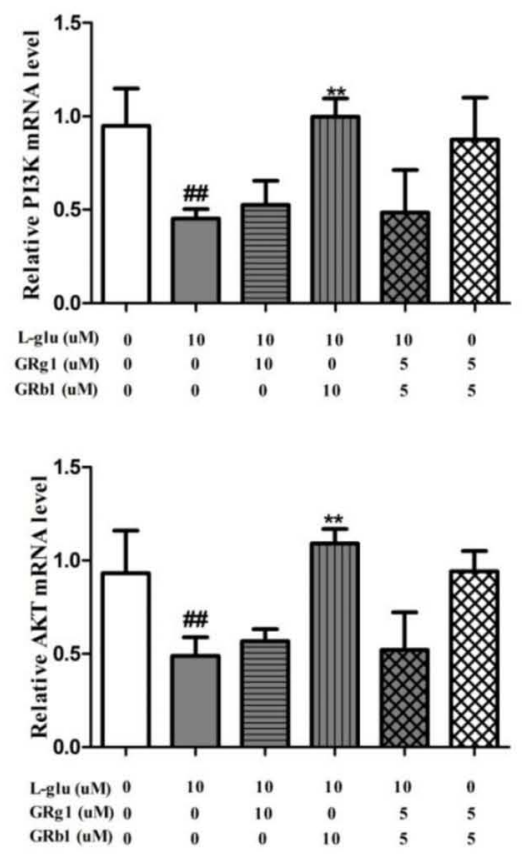

B
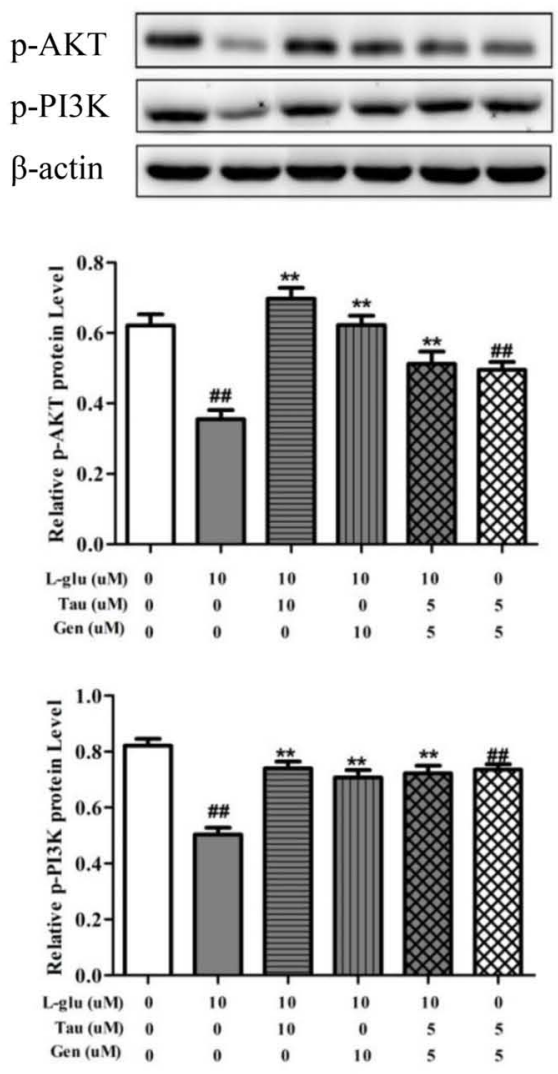
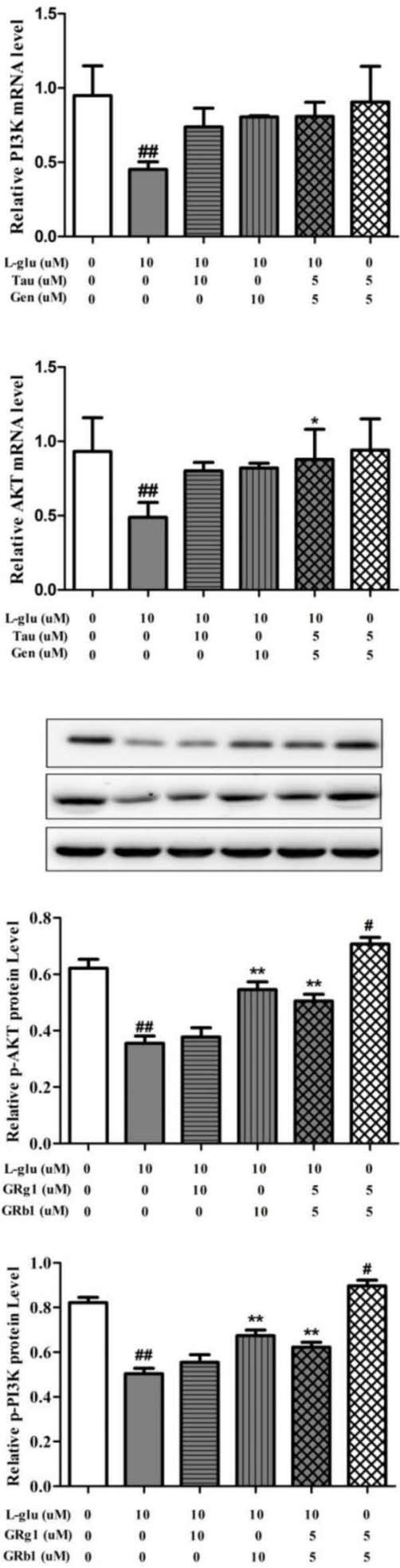

FIGURE 10 | Effects of Taurine, Geniposide, GRg1, and GRb1 in LP on L-Glu-damaged PC12 and normal PC12. (A) The PI3K and AKT mRNA level by RT-QPCR. (B) The $\mathrm{p}$-AKT and $\mathrm{p}$-PI3K protein level. $\beta$-Actin was measured as an internal control. The results are representative of three independent experiments. ${ }^{*} P<0.05$, ${ }^{* *} P<0.01$ vs. L-Glu; ${ }^{\#} P<0.05,{ }^{\#} P<0.01$, vs. control. L-Glu, L-glutamate; Tau, Taurine; Gen, Geniposide; GRg1, Ginsenoside Rg1; GRb1, Ginsenoside Rb1. 
and Geniposide at $5 \mu \mathrm{M}$ down-regulated p-AKT and p-PI3K protein expression on normal PC12. The results showed that Taurine, Geniposide and GRb1 in LP individually up-regulated the level of PI3K and AKT on the level of protein obviously. Furthermore, the combination of Taurine and Geniposide, GRb1 and GRb1 at half-dosage respectively had a significant increase in efficiency on up-regulation of protein expression of PI3K and AKT.

\section{DISCUSSION}

In this study, we have constructed the network pharmacology of LP against ICH based on data-mining and computational modeling. It was performed to investigate biological efficacy of LP for treating ICH in vivo and vitro experiment. Meanwhile we probed into compound combination in LP on L-glutamate-induced PC12 cell. Importantly, our current finding made the complex relation of LP against ICH more unambiguous and provided an insight for understanding the pharmaceutical activity and therapeutic efficacy of LP against $\mathrm{ICH}$.

During the system network construction in mining the relationship between $\mathrm{LP}$ and $\mathrm{ICH}$, the P-T-D network containing 34 potential compounds of LP with146 corresponding targets was obtained. Function of targets of compounds in LP covered inflammation, cell apoptosis, oxidative stress, and neurotransmitters. The network integrally displayed LP had a treatment on $\mathrm{ICH}$ based on multi-compound interaction with multi-target at the molecular level. In the meanwhile, 142 targets of potential compounds were involved in 24 signaling pathways on ICH, Ligand-dependent caspase activation and intrinsic pathway for Apoptosis occupied a large proportion. $\mathrm{ICH}$ has two phases of injury involved. The primary injury emerges from the beginning of bleeding and is primarily due to cerebral vascular injury (Qureshi et al., 2009). Secondary injury might break out with various parallel pathological pathways including excitotoxicity, cytotoxicity of blood, inflammation, blood-brain-barrier disruption, and oxidative stress (Ren et al., 2014). These pathophysiological processes inevitably bring neuronal cell death. PI3K/AKT pathway is a classic pathway which participates in cell survival and proliferation. According to network prediction, we found the PI3K/AKT pathway had close relationship with compounds in LP. Upstream protein PI3K may be regulated by 19 compounds in LP such as Emodin, Baicalein, Ginsenoside Rg1; and downstream protein AKT may be regulated by 17 compounds in LP such as Catalpol, Paeoniflorin, Paeonol, Ginsenoside Rg1.

Hypertension is one of the most common physiological causes of intracerebral hemorrhage. In vivo experiment, we found that LP can decrease levels of HS-CRP, TNF- $\alpha$, NF$\mathrm{k} \beta$ and IL-1 on SHR, which implied LP was capable of inhibiting inflammatory responses on SHR. D2D and E2 levels were also decreased after LP administration, which replied LP could activate fibrinolytic reaction and protect blood vessels. Finally, S-100B and NSE level in SHR were decreased remarkably with treatment of LP. When neurons and axons were disrupted, levels of S-100B and NSE are subsequently released in elevated levels, which possess relatively high specificity and sensitivity for neurological damage (Olivecrona et al., 2015). We concluded that LP overall improved hypertension condition based on the activity of anti-inflammatory, anticoagulation and blood vessel protection. The clonal line PC12 derived from a solid rat adrenal medulla tumor has been used as a dopaminergic neuronal model for in vitro studies. $L$-glutamate obviously inhibited cell proliferation and promoted apoptosis. Pretreatment with LP for $1 \mathrm{~h}$ prior could obviously inhibit apoptosis and promote proliferation, showing that LP protect neuron from amino acid damage during the process of excitotoxicity.

Multi-component combination was a feature of TCM treatment of diseases. From the properties analysis of ingredients in LP, heat-clearing herb PR was different from blood-activating herb RO. Animal ingredient BB was obviously different from herbal ingredient. However, they were used in combination in LP. Effect of their potential compound combination was preliminarily explored on cell proliferation based on factorial design (Xu et al., 2017). Factorial design is a multi-factor cross-group design, which can not only test the differences between the levels of each factor, but also test the interaction between the factors. The result showed that first-level interactions existed and secondary interaction was not found. First-level interaction existed between Taurine and Paeonol, Taurine and Geniposide, Paeonol and Geniposide. Furthermore, GRg1 and GRb1 derived from PN had the interaction. Meanwhile, we found that Taurine and Geniposide, GRg1 and GRb1 had a significant increase in efficiency at the low concentration on inhibiting the apoptosis of PC12 cell; Ginsenoside Rg1 and Ginsenoside $\mathrm{Rb} 1$ at the low concentration had a significant increase in efficiency on the up-regulation expresssion of PI3K and AKT protein. The geniposide and taurine had a $s$ significant increase in efficiency at the low concentration on $\mathrm{PI} 3 \mathrm{~K}$ and $\mathrm{AKT}$ protein when PC12 cell was damaged by glutamate.

This is the first time to study pharmacological effects and therapeutic efficacy of LP on ICH by means of network pharmacology. The research provided new insight for LP against $\mathrm{ICH}$ according to the way of "multi-component, multi-target, multi-pathway. LP played a integrated role on cerebral hemorrhage from anti-inflammatory, anti-coagulation, blood vessel protection and protection neuron from excitotoxicity. Meanwhile, compounds in LP such as Taurine, Geniposide, Ginsenoside Rb1 and Ginsenoside Rg1, were verified that they have intervened in PI3K/AKT pathways. Taurine of BB and Paeonol of PS, GRg1 and GRb1 derived from PN had a $s$ significant increase in efficiency on inhibiting the apoptosis of PC12 cells at the low concentration based on PI3K /AKT pathway.

However, network pharmacology research is a perpetual exploration process based on current level of experiments, Further studies should focus on discovering prototype compounds and metabolic compounds in LP based on pharmacodynamics and pharmacokinetic experiments. 
Interaction of compound includes synergy and antagonism, synergy of multi-component in LP still was needed to systematically study with dose-response in the future. Pathway analysis for targets of potential compounds in LP found a large number of signal pathways was in urgent need of exploring the inner complicated mechanism of LP against ICH.

\section{ETHICS STATEMENT}

Experimental animal ethics committee of Nanjing University of Traditional Chinese Medicine approved the experiment.

Application Number: A171101.

Approval Number: ACU 171103.

\section{REFERENCES}

Biffi, A., Anderson, C. D., Battey, T. W., Ayres, A. M., Greenberg, S. M., Viswanathan, A., et al. (2015). Association between blood pressure control and risk of recurrent intracerebral hemorrhage. JAMA 314, 904-912. doi: 10.1001/ jama.2015.10082

Chen, Y., Li, G. C., Xu, L., Yin, L., Wang, J., Qin, L., et al. (2016). Efficacy and mechanism of Liangxue Tongyu Decoction in the treatment of acute cerebral hemorrhage in rats. J. Tradit. Chin. Med. 33, 683-668.

Chong, B. H., Chan, K. H., Pong, V., Lau, K. K., Chan, Y. H., Zuo, M. L., et al. (2012). Use of aspirin in Chinese after recovery from primary intracranial haemorrhage. Thromb. Haemost. 107, 241-247. doi: 10.1160/TH11-06-0439

Deng, M., Huang, L., Ning, B., Wang, N., Zhang, Q., Zhu, C., et al. (2016). Betaasarone improves learning and memory and reduces acetyl cholinesterase and Beta-amyloid 42 levels in APP/PS1 transgenic mice by regulating Beclin-1dependent autophagy. Brain Res. 1652, 188-194. doi: 10.1016/j.brainres.2016. 10.008

Feigin, V. L., Lawes, C. M., Bennett, D. A., Barker-Collo, S. L., and Parag, V. (2009). Worldwide stroke incidence and early case fatality reported in 56 populationbased studies: a systematic review. Lancet Neurol. 8, 355-369. doi: 10.1016/ s1474-4422(09)70025-0

Huang, X., Li, G. C., Yin, L., Zhang, Z. H., Liang, Y. X., and Chen, H. B. (2015). The effective parts of liangxue tongyu prescription on cooling-blood and activating-blood and analysis of chemical constituents by HPLC-MS and GC-MS. Acta Pharmaceutica Sinica 50, 86-93. doi: 10.16438/j.0513-4870.2015. 01.003

Huang, X. P., Ding, H., Lu, J. D., Tang, Y. H., Deng, B. X., and Deng, C. Q. (2015). Effects of the combination of the main active components of astragalus and panax notoginseng on inflammation and apoptosis of nerve cell after cerebral ischemia-reperfusion. Am. J. Chin. Med. 43, 1419-1438. doi: 10.1142/ s0192415x15500809

Huang, Y., Zhou, X., Zhao, F., Wu, M., Zhou, X., and Wang, M. (2014). Influence of liangxu tongyu fang on expression of cytokines in the peripheral blood of patients with hemorrhagic stroke. Pharmacol. Clin. Chin. Mater. Med. 30, 126-128. doi: 10.13412/j.cnki.zyyl.2014.01.040

Jacquin, M. A., Chiche, J., Zunino, B., Beneteau, M., Meynet, O., Pradelli, L. A., et al. (2013). GAPDH binds to active Akt, leading to Bcl-xL increase and escape from caspase-independent cell death. Cell Death Differ. 20, 1043-1054. doi: $10.1038 / \mathrm{cdd} .2013 .32$

Jiang, B., Shen, R. F., Bi, J., Tian, X. S., Hinchliffe, T., and Xia, Y. (2015). Catalpol: a potential therapeutic for neurodegenerative diseases. Curr. Med. Chem. 22, 1278-1291.

Jin, X., Wang, J., Xia, Z. M., Shang, C. H., Chao, Q. L., Liu, Y. R., et al. (2016). Anti-inflammatory and anti-oxidative activities of paeonol and its metabolites through blocking $\mathrm{MAPK} / \mathrm{ERK} / \mathrm{p} 38$ signaling pathway. Inflammation 39, 434-446. doi: 10.1007/s10753-015-0265-3

Koh, P. O. (2014). Estradiol ameliorates the reduction in parvalbumin expression induced by ischemic brain injury. Neurosci. Lett. 574, 36-40. doi: 10.1016/j. neulet.2014.05.006

\section{AUTHOR CONTRIBUTIONS}

$\mathrm{XL}$ and $\mathrm{XH}$ developed the model. YT calibrated the model. FZ collected compound information in LP. GL and LY provided suggestions and material support. YC reviewed the manuscript.

\section{FUNDING}

This study was sponsored by The Natural Science Foundation of Jiangsu Province of China 2014 (BK20161575 to GL) and the Priority Academic Program Development of Jiangsu Higher Education Institutions (integration of Chinese and Western medicine).

Lipinski, C. A., Lombardo, F., Dominy, B. W., and Feeney, P. J. (1997). Experimental and computational approaches to estimate solubility and permeability in drug discovery and development settings. Adv. Drug Deliv. Rev. 23, 3-25. doi: 10.1016/S0169-409X(96)00423-1

Liu, D. L., Zhao, L. X., Zhang, S., and Du, J. R. (2016). Peroxiredoxin 1-mediated activation of TLR4/NF-kappaB pathway contributes to neuroinflammatory injury in intracerebral hemorrhage. Int. Immunopharmacol. 41, 82-89. doi: 10.1016/j.intimp.2016.10.025

Liu, R., Duan, J. A., Wu, H., Liu, P., Shang, E. X., and Qian, D. W. (2015). Analysis and identification of water soluble components of waterbuffalo horn. Acta Pharmaceutica Sinica 50, 594-598. doi: 10.16438/j.0513-4870.2015. 05.007

Liu, R., Wang, M., and Duan, J. A. (2010). Antipyretic and antioxidant activities of the aqueous extract of Cornu bubali (water buffalo horn). Am. J. Chin. Med. 38, 293-306. doi: 10.1142/s0192415x10007853

Lok, J., Leung, W., Murphy, S., Butler, W., Noviski, N., and Lo, E. H. (2011). Intracranial hemorrhage: mechanisms of secondary brain injury. Acta Neurochir. Suppl. 111, 63-69. doi: 10.1007/978-3-7091-0693-8_11

Ma, H., Guo, W., Feng, Y., Yuan, Y., Xin, Z., and Xiong, L. (2011). Treatment with liangxuetongyu formula in 96 patients with syndrome of stasis-heat obstructing orifices of acute cerebral hemorrhage. Beijing Univ. TCM 34, 348-352.

McNamara, C. R., Ahuja, R., Osafo-Addo, A. D., Barrows, D., Kettenbach, A., Skidan, I., et al. (2013). Akt regulates TNFalpha synthesis downstream of RIP1 kinase activation during necroptosis. PLoS One 8:e56576. doi: 10.1371/journal. pone. 0056576

Min, H., Hong, J., Cho, I. H., Jang, Y. H., Lee, H., Kim, D., et al. (2015). TLR2-induced astrocyte MMP9 activation compromises the blood brain barrier and exacerbates intracerebral hemorrhage in animal models. Mol. Brain 8:23. doi: 10.1186/s13041-015-0116-z

Olivecrona, Z., Bobinski, L., and Koskinen, L. O. (2015). Association of ICP, CPP, CT findings and S-100B and NSE in severe traumatic head injury. Prognostic value of the biomarkers. Brain Inj. 29, 446-454. doi: 10.3109/02699052.2014. 989403

Qureshi, A. I., Mendelow, A. D., and Hanley, D. F. (2009). Intracerebral haemorrhage. Lancet 373, 1632-1644. doi: 10.1016/s0140-6736(09)60371-8

Ren, C., Guingab-Cagmat, J., Kobeissy, F., Zoltewicz, S., Mondello, S., Gao, M., et al. (2014). A neuroproteomic and systems biology analysis of rat brain post intracerebral hemorrhagic stroke. Brain Res. Bull. 102, 46-56. doi: 10.1016/j. brainresbull.2014.02.005

Rodriguez, C., Sobrino, T., Agulla, J., Bobo-Jimenez, V., Ramos-Araque, M. E., Duarte, J. J., et al. (2017). Neovascularization and functional recovery after intracerebral hemorrhage is conditioned by the Tp53 Arg72Pro singlenucleotide polymorphism. Cell Death. Differ. 24, 144-154. doi: 10.1038/cdd. 2016.109

Shannon, P., Markiel, A., Ozier, O., Baliga, N. S., Wang, J. T., Ramage, D., et al. (2003). Cytoscape: a software environment for integrated models of biomolecular interaction networks. Genome Res. 13, 2498-2504. doi: 10.1101/ gr.1239303 
Shi, L., Wang, G., Wei, Y. L., Nan, L. S., Wei, X. Q., and Jie, X. (2017). Therapeutic effect of liangxue tongyu prescrition on 73 cases of cerebral hemorrhage in acute stage. Shangxi Med. J. 46, 583-584. doi: 10.3969/j.issn.0253-9926.2017. 05.035

Steiner, T., Al-Shahi Salman, R., Beer, R., Christensen, H., Cordonnier, C., Csiba, L., et al. (2014). European stroke organisation (ESO) guidelines for the management of spontaneous intracerebral hemorrhage. Int. J. Stroke 9, 840-855. doi: 10.1111/ijs.12309

Taylor, R. A., Chang, C. F., Goods, B. A., Hammond, M. D., Mac Grory, B., Ai, Y., et al. (2017). TGF-betal modulates microglial phenotype and promotes recovery after intracerebral hemorrhage. J. Clin. Invest. 127, 280-292. doi: 10. $1172 /$ jci88647

van Asch, C. J., Luitse, M. J., Rinkel, G. J., van der Tweel, I., Algra, A., and Klijn, C. J. (2010). Incidence, case fatality, and functional outcome of intracerebral haemorrhage over time, according to age, sex, and ethnic origin: a systematic review and meta-analysis. Lancet Neurol. 9, 167-176. doi: 10.1016/s14744422(09)70340-0

Velmurugan, B. K., Yang, H. H., Sung, P. J., and Weng, C. F. (2017). Excavatolide $\mathrm{B}$ inhibits nonsmall cell lung cancer proliferation by altering peroxisome proliferator activated receptor gamma expression and PTEN/AKT/NF-Kbeta expression. Environ. Toxicol. 32, 290-301. doi: 10.1002/tox. 22235

Wang, T., Huang, Y., Zhang, M., Wang, L., Wang, Y., Zhang, L., et al. (2013). [Gly14]-Humanin offers neuroprotection through glycogen synthase kinase-3beta inhibition in a mouse model of intracerebral hemorrhage. Behav. Brain Res. 247, 132-139. doi: 10.1016/j.bbr.2013. 03.023

Wang, Y. H., Chen, K. M., Chiu, P. S., Lai, S. C., Su, H. H., Jan, M. S., et al. (2016). Lumbrokinase attenuates myocardial ischemia-reperfusion injury by inhibiting TLR4 signaling. J. Mol. Cell Cardiol. 99, 113-122. doi: 10.1016/j.yjmcc.2016. 08.004

Wen, Z., Mei, B., Li, H., Dou, Y., Tian, X., Shen, M., et al. (2017). P2X7 Participates in intracerebral hemorrhage-induced secondary brain injury in rats via MAPKs signaling pathways. Neurochem. Res. 42, 2372-2383. doi: 10.1007/s11064-0172257-1
Xu, J. J., Shang, M. Y., Xu, F., Li, Y. L., Liu, G. X., Wang, X., et al. (2017). Comparision and analysis on the blood concentration of common chinese medicine and western medicine. Acta Pharmaceutica Sinica 50, 1222-1234. doi: 10.16438/j.0513-4870.2017-0285

Xu, X., Zhang, W., Huang, C., Li, Y., Yu, H., Wang, Y., et al. (2012). A novel chemometric method for the prediction of human oral bioavailability. Int. J. Mol. Sci. 13, 6964-6982. doi: 10.3390/ijms13066964

Yu, Z. J., Li, J. S., Peng, W., Wang, P., Xiong, L., Liu, Y. J., et al. (2017). Effect of animal medicines for "extinguishing wind to arrest convulsions" on central nervous system diseases. China J. Chi. Mater. Med. 63, 1-10. doi: 10.19540/j. cnki.cjcmm.20171113.015

Zhang, L. K., Gou, W. F., Xu, D., Yuan, Y., Li, J. X., Ma, H. M., et al. (2012). Clinical observation on liangxue tongyu formula combined with western medicine for acute hemorrhagic stroke with the TCM syndrome of stasis-heat obstructing in the brain: a report on 73 Cases. J. Tradit. Chin. Med. 53, 28-30. doi: 10.13288/j. $11-2166 /$ r.2012.01.022

Zhou, J., Du, T., Li, B., Rong, Y., Verkhratsky, A., and Peng, L. (2015). Crosstalk between MAPK/ERK and PI3K/AKT signal pathways during brain ischemia/reperfusion. ASN Neuro 7:1759091415602463. doi: 10.1177/ 1759091415602463

Zhou, Y., Wang, Y., Wang, J., Anne Stetler, R., and Yang, Q. W. (2014). Inflammation in intracerebral hemorrhage: from mechanisms to clinical translation. Prog. Neurobiol. 115, 25-44. doi: 10.1016/j.pneurobio.2013.11.003

Conflict of Interest Statement: The authors declare that the research was conducted in the absence of any commercial or financial relationships that could be construed as a potential conflict of interest.

Copyright (C) 2018 Li, Huang, Tang, Zhao, Cao, Yin and Li. This is an open-access article distributed under the terms of the Creative Commons Attribution License (CC BY). The use, distribution or reproduction in other forums is permitted, provided the original author(s) and the copyright owner(s) are credited and that the original publication in this journal is cited, in accordance with accepted academic practice. No use, distribution or reproduction is permitted which does not comply with these terms. 